\title{
Dependence of twinned volume fraction on loading mode and schmid factor in randomly textured magnesium.
}

Jan Čapek $^{1,2^{*}}$, Kristián Máthis ${ }^{1}$, Bjørn Clausen ${ }^{3}$, Matthew Barnett ${ }^{4}$

${ }^{1}$ Department of Physics of Materials, Faculty of Mathematics and Physics, Charles

University, Ke Karlovu 5, 12116 Prague, Czech Republic

${ }^{2}$ Nuclear Physics Institute of the Academy of Sciences of the Czech Republic, $\check{\text { Rež, }} 250$

68, Czech Republic

${ }^{3}$ Los Alamos National Laboratory, MST-8, Los Alamos, NM 87545, USA

${ }^{4}$ Deakin University, Geelong, 3216, Australia

\begin{abstract}
The twinning activity in random textured cast magnesium during room temperature tension and compression tests was monitored by neutron diffraction (ND). Variation of integrated intensities of selected diffraction peaks, characterizing the twinned volume in particularly oriented grains was compared with calculated Schmid factors for extension twinning of these grains. It is shown that in tension there is a direct proportion between the twinned volume and the maximum value of the Schmid factor for a given grain orientation. In contrast, in compression this relation is not valid. The twinned volume in this case is influenced by both deviation of the grain from the ideal orientation and its rotation around the c-axis. The experimental results are in good agreement with those obtained from elasto-plastic self-consistent (EPSC) modeling.
\end{abstract}

\footnotetext{
* Corresponding author: e-mail: jan.capek89@gmail.com; Phone: +420-221 911 619; Fax: +420-221911 490
} 


\section{Introduction}

$\{10 \overline{1} 2\}\langle 10 \overline{1} 1\rangle$ extension twinning is an important deformation mechanism in magnesium alloys [1-3]. It exhibits polar behavior and differently oriented grains undergo twinning during tensile and compressive deformation. Thus, the plastic behavior and texture evolution depends strongly on the loading direction. There is discussion in the literature [4-8] as to the extent that activation of a given twin variant depends on its Schmid factor (SF). The SF-volume correlation is not necessarily straightforward, since the stresses that drive twinning vary locally and the accommodation of the twinning strain also has to be taken into account $[9,10]$. The common approach for answering this question usually includes statistical analysis of EBSD data. However, this technique is limited in terms of the volume of material that can be examined.

In-situ neutron diffraction has been proved to be useful for investigation of deformation twinning [11-13]. The large penetration depth of the thermal neutrons facilitates the investigation of relatively large sample volumes and provides good statistics also in the case of coarse-grained $(>50 \mu \mathrm{m})$ materials. The information about twinning can be obtained by following the intensity changes of particular diffraction peaks $[11,14,15]$.

In this work we employ the neutron diffraction method to study the Schmid factor dependence of twinning. Correspondence between the experimentally established twinned volume fraction and the calculated Schmid factors for extension twinning in 
particular orientation families is analyzed ${ }^{1}$. The experimental data, measured on randomly textured $\mathrm{Mg}-\mathrm{Al}$ alloy are compared with predictions of an EPSC model.

\section{Material and experimental methods}

Binary $\mathrm{Mg}-2$ wt. \% $\mathrm{Al}(\mathrm{Mg} 2 \% \mathrm{Al})$ alloy having a grain size of approximately $100 \mu m$ was used for the experiment[16]. The testing was carried out using cylindrical specimens with a diameter of $9 \mathrm{~mm}$ and gauge length of $20 \mathrm{~mm}$. Inverse pole figures show that the initial samples have a random grain orientation distribution (Fig. 1). Monotonic compression and tensile tests were performed at room temperature at a strain rate of $10^{-3} \mathrm{~s}^{-1}$. The test was stopped for approx. $70 \mathrm{~min}$ at particular true strain values $(0.1 \%, 0.5 \%, 1 \%, 2 \%, 3 \%, 4 \%, 5 \%$, and $6 \%)$ in order to collect the neutron diffraction data. The SMARTS engineering instrument at LANSCE was used for collecting the diffraction pattern [17]. The diffraction patterns were measured using two detector banks at $\pm 90^{\circ}$ to the incident beam. The angle between the incident beam and the loading direction was $45^{\circ}$. This setup provides diffraction measurement of crystallographic planes both perpendicular (axial detector) and parallel (transversal detector) to the loading direction. We evaluated data from the axial detector which shows the grain orientation with respect to the loading direction. The measured data were fitted by EPSC model in order to better understand the changes in peak intensity.

Direct observations of the microstructure of the specimen deformed to $1 \%$ strain were performed by scanning electron microscope FEI Quanta 200F. The electron backscatter diffraction (EBSD) was used to study of the activated twin variants. The specimens for EBSD investigations were polished by standard methods down to OPS

\footnotetext{
${ }^{1}$ Contraction $\{10 \overline{1} 1\}$ twins were not considered due to the low strain levels achieved
} 
colloidal silica suspension. The final electropolishing was done by means of Struers AC-2-II electrolyte.

\section{Results}

Examples of the microstructure after $1 \%$ deformation in a) tension; b) compression are shown in Fig 2. The loading direction in both cases is perpendicular to the figure plane. It is obvious that the number of twin variants and size of twins depends significantly on the loading mode. Grains, designated as T1 and C1, are almost ideally oriented for extension twinning in tension and compression, respectively. Several twin variants, having moderate thickness can be observed in grain $\mathrm{T} 1$. In contrast, grain $\mathrm{C} 1$ includes only one twin variant, which has reoriented almost half of the parent grain. In the grains less favorably oriented for twinning (T2, C2), the number of twin variants is larger for the compression case than for tension.

\subsection{Neutron diffraction experiments}

The overall twin volume fraction (TVF) during the deformation has been determined from the diffraction data by performing a 2-bank Rietveld refinement assuming an axisymmetric texture, as presented in [18]. Axisymmetric textures can be represented using the axial distribution function (ADF), which is a cut through the pole figure at a line from the center to the perimeter. From the ADF, one can calculate the twin volume fraction (see Fig. 3) by integrating the area under the ADF [18]. Fig. 3 demonstrates that above $1 \%$ of applied strain the TVF is larger in compression by $\sim 25 \%$. 
The intensity changes for selected parent peaks, which are directly proportional to the TVF [11], are shown in Fig. 4. The selected grain orientation families and the corresponding maximal value of the Schmid factor for extension twinning are listed in Table 1.

Table 1. Selected grain orientations and their maximal SF for tension and compression, respectively.

\begin{tabular}{|c|c|c|c|c|c|c|c|}
\hline \multicolumn{7}{|c|}{ Tension } & \\
\hline Reflection & 0002 & $10 \overline{14}$ & $10 \overline{13}$ & $11 \overline{2} 4$ & $10 \overline{1} 2^{2}$ & $20 \overline{2} 3^{2}$ & \\
\hline $\mathrm{SF}_{\max }$ & 0.50 & 0.40 & 0.34 & 0.30 & 0.22 & 0.14 & \\
\hline \multicolumn{8}{|c|}{ Compression } \\
\hline Reflection & $10 \overline{10}$ & $20 \overline{2} 1$ & $21 \overline{3} 0$ & $21 \overline{3} 1$ & $11 \overline{2} 0^{2}$ & $10 \overline{1} 1^{2}$ & $11 \overline{2} 2^{2}$ \\
\hline $\mathrm{SF}_{\max }$ & 0.50 & 0.45 & 0.45 & 0.42 & 0.37 & 0.31 & 0.16 \\
\hline
\end{tabular}

It is obvious that for tension (Fig. 4a) the twinned volume fraction decreases proportionally with the decreasing value of maximal SF. In compression (Fig. 4b), this dependence is less clear. Many grain orientations behave similarly to the favorably oriented $\{10 \overline{1} 0\}$ reflection, i.e. these grains have almost the same twinned volumes. However, grains with orientations $\{10 \overline{1} 1\}$ and $\{11 \overline{2} 2\}$ exhibit significantly lower twinned volume.

Figure 5 shows a comparison between tension and compression for grain families with similar maximum SF. It can be seen that in some cases the curves overlap but in others they do not. The difference between tension and compression is clearly

\footnotetext{
${ }^{2}$ These peaks may include twin daughters, but their nucleation is highly improbable due to the low SF
} 
orientation dependent. Elucidation of the physical background of this behavior is presented in what follows.

\section{Discussion}

The present results raise three interesting questions:

- Why is the overall twinned volume fraction larger in compression than in tension?

- Why is there a direct proportion between the maximum SF and the twinned volume only in the tension? Further, why do some grains, having almost the same maximum SF, exhibit similar twinned volumes in both tension and compression, while others do not?

- Can EPSC modeling capture these observations?

\subsection{Loading mode dependence of the overall twin volume}

Using the calculations listed in the appendix a statistical distribution of SF in a randomly oriented polycrystal can be determined (Fig.6). It is obvious that in tension there is larger fraction of grains having a positive SF. However, the majority of them have low SF. Only $10 \%$ of grains in tension have SF $>0.4$, while the same criterion is met by $25 \%$ of grains in compression. We can also observe decreasing numbers of grains with increasing SF value in tension and increasing number of grains with increasing SF value in compression. The median value of SF is 0.10 in tension and 0.24 in compression. 
As reported elsewhere [5, 19], high SF twins tend to be thicker than those with low SF, because the driving force for twin boundary migration scales linearly with the SF [20]. Thus, the larger overall twin volume in compression can be elucidated in terms of a larger number of grains with a high SF for twinning.

\subsection{Dependence of the twinned volume fraction on the Schmid factor for extension twinning}

In tension, the TVF for a given orientation monotonically decreases with the deviation angle from the ideal orientation. Further, it is obvious from Fig 7, that there is a linear dependence of the twinned volume on the maximal value of SF for a given orientation. The interpretation of compression data is more difficult. The correlation between maximal SF and twinned volume is not as strong. We see a higher correlation when we plot TVF against average SF (Fig. 8). This reflects the importance of the number of high SF systems in each orientation. For example, it can be seen in Tables 2 and 3 that all six twin systems have $\mathrm{SF}=0.5$ for $(0002)$ in tension but for $\{10 \overline{1} 0\}$ in compression only two systems have $\mathrm{SF}=0.5$. The number of favorably oriented systems within a grain is clearly an important determinant of the TVF, not just the value of the SF. This can explain why, despite similar values of maximal SFs, $\{10 \overline{1} 0\}$ orientations in compression display lower TVFs than (0002) in tension (Fig. 5a).

Table 2. SF for all six twin variants in tension.

\begin{tabular}{|c|c|c|c|c|c|c|}
\hline Reflection & $\mathbf{0 0 0 2}$ & $\mathbf{1 0 \overline { 1 } 4}$ & $\mathbf{1 0 \overline { 1 } 3}$ & $\mathbf{1 1 \overline { 2 } 4}$ & $\mathbf{1 0 \overline { 1 } 2}$ & $\mathbf{2 0 \overline { 2 } 3}$ \\
\hline$\Delta \theta$ & $0^{\circ}$ & $25^{\circ}$ & $32^{\circ}$ & $39^{\circ}$ & $43^{\circ}$ & $51^{\circ}$ \\
\hline
\end{tabular}




\begin{tabular}{|c|c|c|c|c|c|c|}
\hline$\phi$ & $0^{\circ}$ & $0^{\circ}$ & $0^{\circ}$ & $30^{\circ}$ & $0^{\circ}$ & $0^{\circ}$ \\
\hline$(10 \overline{1} 2)$ & 0.50 & 0.30 & 0.19 & 0.13 & 0.00 & -0.14 \\
\hline$(1 \overline{1} 02)$ & 0.50 & 0.37 & 0.31 & 0.30 & 0.19 & 0.10 \\
\hline$(0 \overline{1} 12)$ & 0.50 & 0.40 & 0.34 & 0.17 & 0.22 & 0.13 \\
\hline$(\overline{1} 012)$ & 0.50 & 0.34 & 0.24 & 0.17 & 0.06 & -0.08 \\
\hline$(\overline{1} 102)$ & 0.50 & 0.40 & 0.34 & 0.30 & 0.22 & 0.13 \\
\hline$(01 \overline{1} 2)$ & 0.50 & 0.37 & 0.31 & 0.12 & 0.19 & 0.10 \\
\hline
\end{tabular}

Table 3. SF for all six twin variants in compression.

\begin{tabular}{|c|c|c|c|c|c|c|c|}
\hline Reflection & $\mathbf{1 0} \overline{\mathbf{1}} \mathbf{2 0}$ & $\mathbf{2 0} \mathbf{1}$ & $\mathbf{2 1 3} \mathbf{2 1}$ & $\mathbf{2 1} \mathbf{1 1 2} 0$ & $\mathbf{1 0} \overline{\mathbf{1}}$ & $\mathbf{1 1 2} 2$ \\
\hline$\Delta \theta$ & $0^{\circ}$ & $15^{\circ}$ & $0^{\circ}$ & $11^{\circ}$ & $0^{\circ}$ & $28^{\circ}$ & $31^{\circ}$ \\
\hline$\phi$ & $0^{\circ}$ & $0^{\circ}$ & $19^{\circ}$ & $19^{\circ}$ & $30^{\circ}$ & $0^{\circ}$ & $30^{\circ}$ \\
\hline$(10 \overline{1} 2)$ & 0.50 & 0.45 & 0.45 & 0.42 & 0.37 & 0.30 & 0.16 \\
\hline$(1 \overline{1} 02)$ & 0.13 & 0.09 & 0.02 & 0.00 & 0.00 & 0.00 & -0.14 \\
\hline$(0 \overline{1} 12)$ & 0.13 & 0.08 & 0.29 & 0.25 & 0.37 & -0.02 & 0.11 \\
\hline$(\overline{1} 012)$ & 0.50 & 0.42 & 0.45 & 0.40 & 0.37 & 0.26 & 0.11 \\
\hline$(\overline{1} 102)$ & 0.13 & 0.08 & 0.02 & 0.00 & 0.00 & -0.02 & -0.14 \\
\hline$(01 \overline{1} 2)$ & 0.13 & 0.09 & 0.29 & 0.26 & 0.37 & 0.00 & 0.16 \\
\hline
\end{tabular}

According to [4], the nucleation of an additional twin variant in a grain besides that having the highest SF depends on the ratio of SFi/SFmax. We have calculated SF for all 6 twin variants (see [13]) and the ratio between the best variant and the others (see IPF in the appendix Fig.B1). These IPFs show a completely different behavior in 
compression. The effect is clear in Tables 2 and 3. In compression, the SF of $3^{\text {rd }}$ ranked system is always less than 0.4 , the $5^{\text {th }}$ twin variant is always less than 0.2 . In compression the probability of activation of all six twin variants within a grain will be very low. We can thus conclude that in tension the probability of activation of multiple twin variants within a grain is considerably higher than in compression. If we plot the number of variants with SF higher than $90 \%$ of the best variant (Fig.9) against maximal SF, we can observe this difference quite clearly. In tension, the high SF grains have the highest number of high SF variants. On the contrary, in compression, there is no direct correlation between number of twin variants and maximal SF.

In Figure 5, the orientation pairs $\{10 \overline{1} 0\}_{C}-(0002)_{T}$ and $\{10 \overline{1} 1\}_{C}-\{11 \overline{2} 4\}_{T}$, how larger TVF in tension. These two pairs show different number of high SF variants

in Figure 9. In case of the other orientation pairs in Figure $5\{11 \overline{2} 0\}_{C}-\{10 \overline{1} 3\}_{T}$, $\{21 \overline{3} 1\}_{C}-\{10 \overline{1} 4\}_{T}$ and $\{11 \overline{2} 2\}_{C}-\{20 \overline{2} 3\}_{T}$ the TVF is similar. These pairs show similar numbers of high SF variants in Figure 9. Thus, the different behavior of TVF dependence on SF is due to the difference in the number of high SF variants. In tension, with increasing $\Delta \theta$ both the value of $\mathrm{SF}_{\max }$ and the number of high SF twin variants proportionally decrease. In compression, this dependence is not straightforward, since not only the deviation from the ideal orientation but also the rotation around the c-axis $(\phi)$ is an important parameter.

\subsection{EPSC modeling}

Finally, the deformation behavior was modeled using an Elasto-Plastic Self-Consistent (EPSC) model to verify that it could capture the present findings [18]. The reason for 
that was the capability of the EPSC for predicting the number of twin variants, which are nucleated within a particular grain.

The Voce parameters used for fitting are shown in Table 4. The match between the experimental data and the theoretical curve is satisfying (Fig. 10).

Table 4. Voce parameters for compression test

\begin{tabular}{|l|r|r|r|r|}
\hline & $\tau_{0}[M P a]$ & $\tau_{1}[M P a]$ & $\theta_{0}[M P a]$ & $\theta_{1}[M P a]$ \\
\hline Basal & 13 & 7 & 100 & 50 \\
\hline Prismatic & 25 & 5 & 120 & 15 \\
\hline 2nd order pyramidal & 120 & 90 & 200 & 100 \\
\hline Twinning & 8 & 0 & 0 & 0 \\
\hline
\end{tabular}

The EPSC model gives further information about the texture changes, which can be utilized for calculation of theoretical diffraction patterns. From the output of the EPSC we calculated the relative intensity changes of diffraction peaks measured also by neutron diffraction (see above). It is evident from Fig. 11 that the EPSC model predicts similar behavior shown in Figure 4.

Our assumption is that nucleation of more variants that provides different strain direction is an important parameter for twinned volume. Twin variants (1012)[1]011]

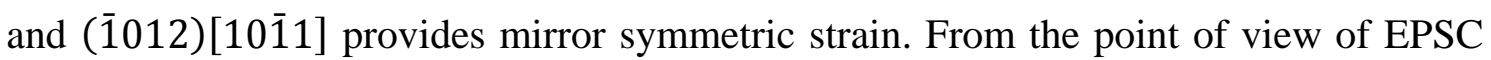
model there is not a big difference between them and they can be considered together. Similarly, we can make pairs from the other four variants. Therefore, we have 3 variant pairs with different straining. Figs. 12 display the orientations of those grains, which 
according to the EPSC model undergo twinning. The colors represent number of nucleated twin variants within the grains having a particular orientation, predicted by the model. The calculations clearly illustrate that there is a significant difference between the tension and compression case, respectively. And the EPSC modeling results are in excellent agreement with the theoretical calculations presented in Fig. 9.

In Figure 12a the data for tension show for $\Delta \theta<30^{\circ}$ (red area) grains that exhibit nucleation of at least three variants. For $\Delta \theta>30^{\circ}$ nucleation of only two (blue area) or one variant (green area) is observed.

In compression (Figure 12b), at most two variants are predicted to form. The number of variants depends on the $\phi$ angle, this is on the rotation of the grain around the $c$-axis.

The EBSD observations support these conclusions. The presence of all six variants can be observed in grain $\mathrm{T} 1$ in Fig. 6. The orientation of $\mathrm{T} 1$ grain is almost ideal for extension twinning in tension, (SF: 0.46, 0.48, 0.48, 0.48, 0.49, 0.47). However, we can also observe twin nucleation in grains, which are highly deviated from ideal orientation. The deviation of grain T2 (Fig. 2a) is $\theta=77^{\circ}, \phi=25^{\circ}$ and SF in tension are $(-0.35$, $0.28, \mathbf{0 . 0 2},-0.37,-0.30, \mathbf{0 . 0 2})$. Despite the low SF, the EBSD proved nucleation of the variants with positive SF. The twinned volume in this particular grain is not high, but owing to the high number of such oriented grains, their contribution to the overall TVF is not negligible. In contrast, grain $\mathrm{C} 1$ is almost ideally oriented for twinning in compression

(SF: $0.41,0.09,0.06,0.44,0.11,0.05)$. However, the SFi/SFmax ratio for $\mathrm{i}>2$ is small. Consequently, nucleation of two variants (more precisely a pair of twin variants) is observed - exactly, as the EPSC model predicts. The C2 grain is deviated by the 
$\phi=25^{\circ}$ (SF: $\left.0.29,0.22,-0.05,0.32,0.25,-0.06\right)$. All four high SF variants are nucleated which is again in good agreement with the EPSC results.

We thus conclude that the results of EPSC modeling are in good agreement with measured data and theoretical results. The EPSC well predict the anomalous SF dependence of TVF and provides better insight on twin variants selection.

The analysis presented above demonstrates, how the twinned volume fraction can depend on the SF. However, the twin nucleation and growth are influenced also by other microstructural parameters as e.g. the orientation of the neighboring grains [21], stress gradients across the grain [5] or interaction of twin boundaries with dislocations [22]. In order to get a complex picture about the twinning in magnesium alloys, these factors have to be taken also into account. However, such analysis is out of scope of the present paper.

\section{Conclusion}

The evolution of the $\{10 \overline{1} 2\}$ extension twinning during tension and compression testing of Mg-2 wt.\% Al alloy was studied in-situ by neutron diffraction measurements and theoretically by elasto-plastic self-consistent modeling. The following conclusions may be drawn:

- The overall twinned volume is larger in compression owing to the larger number of grains favorably oriented for nucleation of twins with high Schmid factor for twinning,

- In tension, the twinned volume fraction decreases with increasing deviation of the given grain from the ideal orientation for extension twinning.

- In compression, not only the deviation from the ideal orientation, but also the grain rotation around the c-axis plays an important role. The latter parameter 
determines the probability of the activation of further twin variants. Therefore, the ideally oriented and deviated, but properly rotated grains can exhibit the same twinned volume, owing to the higher number of twin variants nucleated in the latter grains.

\section{Acknowledgements}

- The authors are grateful for the financial support of the Czech Science Foundation under the contract 14-36566G. JČ acknowledges the support by the Charles University in Prague, Faculty of Mathematics and Physics, project GA UK Nr. 251715. This work has benefited from the use of the Lujan Neutron Scattering Center at LANSCE, funded by the US Department of Energy's Office of Basic Energy Sciences. Los Alamos National Laboratory is operated by Los Alamos National Security LLC under US DOE Contract DE-AC52-06NA25396. This work was supported by the project "Nanomaterials centre for advanced applications", Project No. CZ.02.1.01/0.0/0.0/15_003/0000485, financed by ERDF.

\section{References}

[1] A. Chapuis, J.H. Driver, Temperature dependency of slip and twinning in plane strain compressed magensium single crystals. Acta Mater 59 (2011) 1986-1994.

[2] M.M. Avedesian, H. Baker, Magnesium and Magnesium Alloys (ASM Specialty Handbook), ASM International, 1999.

[3] J.W. Christian, S. Mahajan, Deformation Twinning. Prog Mater Sci 39 (1995) 1-157.

[4] M.R. Barnett, Z. Keshavarz, A.G. Beer, X. Ma, Non-Schmid behaviour during secondary twinning in a polycrystalline magnesium alloy. Acta Mater 56 (2008) 5-15.

[5] I.J. Beyerlein, L. Capolungo, P.E. Marshall, R.J. McCabe, C.N. Tome, Statistical analyses of deformation twinning in magnesium. Philosophical Magazine 90 (2010) 2161-2190. 
[6] X.-L. Nan, H.-Y. Wang, L. Zhang, J.-B. Li, Q.-C. Jiang, Calculation of Schmid factors in magnesium: Analysis of deformation behaviors. Scripta Mater 67 (2012) 443-446.

[7] C. Guo, R. Xin, C. Ding, B. Song, Q. Liu, Understanding of variant selection and twin patterns in compressed $\mathrm{Mg}$ alloy sheets via combined analysis of Schmid factor and strain compatibility factor. Mat Sci Eng a-Struct 609 (2014) 92-101.

[8] A. Chapuis, Y. Xin, X. Zhou, Q. Liu, $\{10-12\}$ Twin variants selection mechanisms during twinning, re-twinning and detwinning. Materials Science and Engineering: A 612 (2014) 431-439.

[9] F. Wang, S. Sandlöbes, M. Diehl, L. Sharma, F. Roters, D. Raabe, In situ observation of collective grain-scale mechanics in Mg and Mg-rare earth alloys. Acta Mater 80 (2014) 77-93.

[10] K.D. Molodov, T. Al-Samman, D.A. Molodov, G. Gottstein, On the role of anomalous twinning in the plasticity of magnesium. Acta Mater 103 (2016) 711-723.

[11] M.A. Gharghouri, G.C. Weatherly, J.D. Embury, J. Root, Study of the mechanical properties of Mg-7.7at.\% Al by in-situ neutron diffraction. Philos Mag A 79 (1999) 1671-1695.

[12] O. Muransky, M.R. Barnett, D.G. Carr, S.C. Vogel, E.C. Oliver, Investigation of deformation twinning in a fine-grained and coarse-grained ZM20 Mg alloy: Combined in situ neutron diffraction and acoustic emission. Acta Mater 58 (2010) 1503-1517.

[13] J. Čapek, K. Máthis, B. Clausen, J. Straska, P. Beran, P. Lukáš, Study of the loading mode dependence of the twinning in random textured cast magnesium by acoustic emission and neutron diffraction methods. Materials Science and Engineering a-Structural Materials Properties Microstructure and Processing 602 (2014) 25-32.

[14] S.R. Agnew, D.W. Brown, C.N. Tome, Validating a polycrystal model for the elastoplastic response of magnesium alloy AZ31 using in situ neutron diffraction. Acta Mater 54 (2006) 4841-4852.

[15] O. Muransky, D.G. Carr, P. Sittner, E.C. Oliver, In situ neutron diffraction investigation of deformation twinning and pseudoelastic-like behaviour of extruded AZ31 magnesium alloy. Int. J. Plast. 25 (2009) 1107-1127.

[16] K. Mathis, J. Capek, B. Clausen, T. Krajnak, D. Nagarajan, Investigation of the dependence of deformation mechanisms on solute content in polycrystalline $\mathrm{Mg}-\mathrm{Al}$ magnesium alloys by neutron diffraction and acoustic emission. J Alloy Compd 642 (2015) 185-191.

[17] M.A.M. Bourke, D.C. Dunand, E. Ustundag, SMARTS - A spectrometer for strain measurement in engineering materials. Applied Physics A: Materials Science and Processing 74 (2002) S1707-S1709.

[18] B. Clausen, C.N. Tome, D.W. Brown, S.R. Agnew, Reorientation and stress relaxation due to twinning: Modeling and experimental characterization for Mg. Acta Mater 56 (2008) 2456-2468.

[19] A.L. Oppedal, H. El Kadiri, C.N. Tomé, G.C. Kaschner, S.C. Vogel, J.C. Baird, M.F. Horstemeyer, Effect of dislocation transmutation on modeling hardening mechanisms by twinning in magnesium. Int. J. Plast. 30-31 (2012) 41-61.

[20] F. Siska, L. Stratil, J. Cizek, A. Ghaderi, M. Barnett, Numerical analysis of twin thickening process in magnesium alloys. Acta Mater 124 (2017) 9-16.

[21] M.R. Barnett, N. Stanford, A. Ghaderi, F. Siska, Plastic relaxation of the internal stress induced by twinning. Acta Mater 61 (2013) 7859-7867.

[22] H. El Kadiri, J.C. Baird, J. Kapil, A.L. Oppedal, M. Cherkaoui, S.C. Vogel, Flow asymmetry and nucleation stresses of twinning and non-basal slip in magnesium. Int. J. Plast. 44 (2013) 111-120. 


\section{Appendix}

A. Calculation of the loading mode dependence of the Schmid factors for extension twinning (Fig A.1)

Generally, the Schmid factor, giving proportion between the critically resolved shear stress $(\tau)$ on a slip plane and the stress applied to the material $(\sigma)$ is given by:

$$
m=\cos \varphi \cos \lambda
$$

where $\varphi$ is angle between loading direction $(\vec{F})$ and slip plane normal $(\vec{n})$ and $\lambda$ is angle between loading direction and slip direction $(\vec{S})$.

We did the calculation in Cartesian coordinates system selecting the loading direction aligns with the $z$-axis $\left(\vec{F}=|F| \overrightarrow{e_{z}}\right)$ and using Euler angles and Bunge notation $\left(\phi_{1} \theta \phi_{2}\right)$ to define the grain orientation. In the initial state configuration $\left(0^{\circ} 0^{\circ} 0^{\circ}\right)$ the basal plane (0001) is perpendicular to $z$-axis and prismatic plane $(10 \overline{10})$ is perpendicular to the $y$-axis. Therefore the $\theta$ rotation is around the $x$-axis, which is the $\langle 1 \overline{2} 10\rangle$ direction. The $\phi_{1}$ rotation takes place around the $z$ axis (=loading direction). Thus, it does not influence the angle between loading direction and slip direction. Therefore and we can set it as $0^{\circ}$ and simplify an expression for Euler angles as $(\theta \phi)$. Using the vector algebra, we can write the Schmid law as follows:

$$
m=\left(\overrightarrow{e_{n}}(\theta \phi) \cdot \overrightarrow{e_{z}}\right)\left(\overrightarrow{e_{s}}(\theta \phi) \cdot \overrightarrow{e_{z}}\right)
$$

There are six twinning planes and each of them has one twinning direction. The following calculation is done for the (10 $\overline{1} 2)[10 \overline{1} \overline{1}]$ variant. The $\phi$ angle for the other variants can be expressed as $\phi=\phi^{\prime}+k \cdot 60^{\circ}$, where $k \in\{0,1,2,3,4,5\}$. The plane 
normal vector and slip direction are expressed as a combination of the initial state and the rotation matrix.

$$
\begin{aligned}
& \vec{n}=(0, c / a, \sqrt{3}) \\
& \vec{s}=(0,-\sqrt{3}, c / a)
\end{aligned}
$$

Since we needn't take into account the $\phi_{1}$ rotation, there are only two rotation matrices.

$$
\begin{aligned}
C(\theta) & =\left(\begin{array}{ccc}
1 & 0 & 0 \\
0 & \cos \theta & \sin \theta \\
0 & -\sin \theta & \cos \theta
\end{array}\right) \\
B(\phi) & =\left(\begin{array}{ccc}
\cos \phi & \sin \phi & 0 \\
-\sin \phi & \cos \phi & 0 \\
0 & 0 & 1
\end{array}\right)
\end{aligned}
$$

The transformation from the coordinate system connected with the crystal to base fixed to the loading direction is:

$$
\begin{aligned}
& \vec{n}(\theta \phi)=(B(\phi) C(\theta))^{-1} \overrightarrow{n_{0}} \\
& (B(\phi) C(\theta))^{-1}=\left(\begin{array}{ccc}
\cos \phi & -\sin \phi & 0 \\
\cos \theta \sin \phi & \cos \theta \cos \phi & -\sin \theta \\
\sin \theta \sin \phi & \cos \phi \sin \theta & \cos \theta
\end{array}\right)
\end{aligned}
$$

From eq. (A.2)-(A.8) we get:

$$
m=\left((B(\phi) C(\theta))^{-1} \overrightarrow{e_{n_{0}}} \cdot \overrightarrow{e_{z}}\right)\left((B(\phi) C(\theta))^{-1} \overrightarrow{e_{s_{0}}} \cdot \overrightarrow{e_{z}}\right)
$$

Since $\overrightarrow{e_{z}}=(0,0,1)$, we are only interested about the $z$ projection of $\overrightarrow{e_{n}}, \overrightarrow{e_{s}}$.

$$
m=\frac{1}{(c / a)^{2}+3}(\cos \phi \sin \theta c / a+\cos \theta \sqrt{3})(-\cos \phi \sin \theta \sqrt{3}+\cos \theta c / a)
$$

Using the relation between trigonometric functions

$$
\cos \left(\phi+60^{\circ}\right)=(\cos \phi-\sqrt{3} \sin \phi) / 2 \text { and } \cos \left(\phi+120^{\circ}\right)=(-\cos \phi-\sqrt{3} \sin \phi) / 2
$$
we can calculate SF for all variants.

$\theta$ - angle between the $c$-axis and the loading direction 
$\phi$ - is the angle describing rotation of the unit cell around the c-axis

\begin{tabular}{|l|c|c|c|c|c|c|}
\hline SF & $m_{t w}^{1}$ & $m_{t w}^{2}$ & $m_{t w}^{3}$ & $m_{t w}^{4}$ & $m_{t w}^{5}$ & $m_{t w}^{6}$ \\
\hline Twinning plane & $(10 \overline{1} 2)$ & $(1 \overline{1} 02)$ & $(0 \overline{1} 12)$ & $(\overline{1} 012)$ & $(\overline{1} 102)$ & $(01 \overline{1} 2)$ \\
\hline
\end{tabular}

Table A1. Assignment of SF to the twinning plane

$m_{t w}^{1}=(3 \cos \theta+2 \sqrt{2} \sin \theta \cos \phi)(4 \sqrt{2} \cos \theta-6 \sin \theta \cos \phi) / 34$

$m_{t w}^{2}=$

$(3 \cos \theta+\sqrt{2} \sin \theta(\cos \phi-\sqrt{3} \sin \phi))(4 \sqrt{2} \cos \theta-3 \sin \theta(\cos \phi-\sqrt{3} \sin \phi)) / 34$

$m_{t w}^{3}=$

$(3 \cos \theta-\sqrt{2} \sin \theta(\cos \phi+\sqrt{3} \sin \phi))(4 \sqrt{2} \cos \theta+3 \sin \theta(\cos \phi+\sqrt{3} \sin \phi)) / 34$

$m_{t w}^{4}=(3 \cos \theta-2 \sqrt{2} \sin \theta \cos \phi)(4 \sqrt{2} \cos \theta+6 \sin \theta \cos \phi) / 34$

$m_{t w}^{5}=$

$(3 \cos \theta-\sqrt{2} \sin \theta(\cos \phi-\sqrt{3} \sin \phi))(4 \sqrt{2} \cos \theta+3 \sin \theta(\cos \phi-\sqrt{3} \sin \phi)) / 34$

$m_{t w}^{6}=$

$(3 \cos \theta+\sqrt{2} \sin \theta(\cos \phi+\sqrt{3} \sin \phi))(4 \sqrt{2} \cos \theta-3 \sin \theta(\cos \phi+\sqrt{3} \sin \phi)) / 34$

(A.16)

B. Calculation of the ratio between the best variant and the others.

SF of coupled variants $(1-4,2-5,3-6)$ are similar, we will work further only with the variant with higher SF. In compression $m_{t w}^{1}>m_{t w}^{6}>m_{t w}^{2}$, in tension $m_{t w}^{5}>$ $m_{t w}^{3}>m_{t w}^{4}$ 
We plotted the IPF of SF for the best variant and ratio between others variants and the best one (Fig. B.1). 


\section{List of figure caption}

Figure 1. Inverse pole figure of $\mathrm{Mg} 2 \% \mathrm{Al}$ tension sample in axial (loading) direction measured using neutron diffraction.

Figure 2. EBSD maps of the microstructure after $1 \%$ strain in a) tension; b) in compression. Loading direction is perpendicular to the figures' planes.

Figure 3. Measured twin volume fraction for tensile and compressive loading to $6 \%$ strain.

Figure 4. Twin volume fraction of selected grain families in a) tension, b) compression.

Figure 5. Relative intensity changes for grains with similar SF.

Figure 6. Fraction of grains having a particular Schmid factor for extension twinning, calculated for a randomly textured $\mathrm{Mg}$ polycrystal

Figure 7. Dependence of twinned volume fraction at 3\% deformation on the maximal Schmid factor for extension twinning

Figure 8. Dependence of twinned volume fraction at 3\% deformation on average Schmid factor

Figure 9. Dependence of number of twin variants with SF value higher than $90 \%$ of the maximal value on the maximal value of SF

Figure 10. Measured and fitted deformation curves a) tension b) compression

Figure 11. Diffraction peak intensity changes in selected oriented grains for twinning in a) tension b) compression

Figure 12. EPSC analysis of twin variant selection in a) tension b) compression

Figure 13. EPSC analysis of twin variant selection in compression

Figure A.1 Variation of the Schmid factors for extension twinning in a) tension, b) compression in $\theta-\phi$ space calculated using Eqs. 11-16. The blue color represents negative values.

Figure B.1 IPF of Schmid factor for a) tension b) compression and ratio of Schmid factors between the c,d) $3^{\text {rd }}$, resp. e,f) $5^{\text {th }}$ variant and the best one 


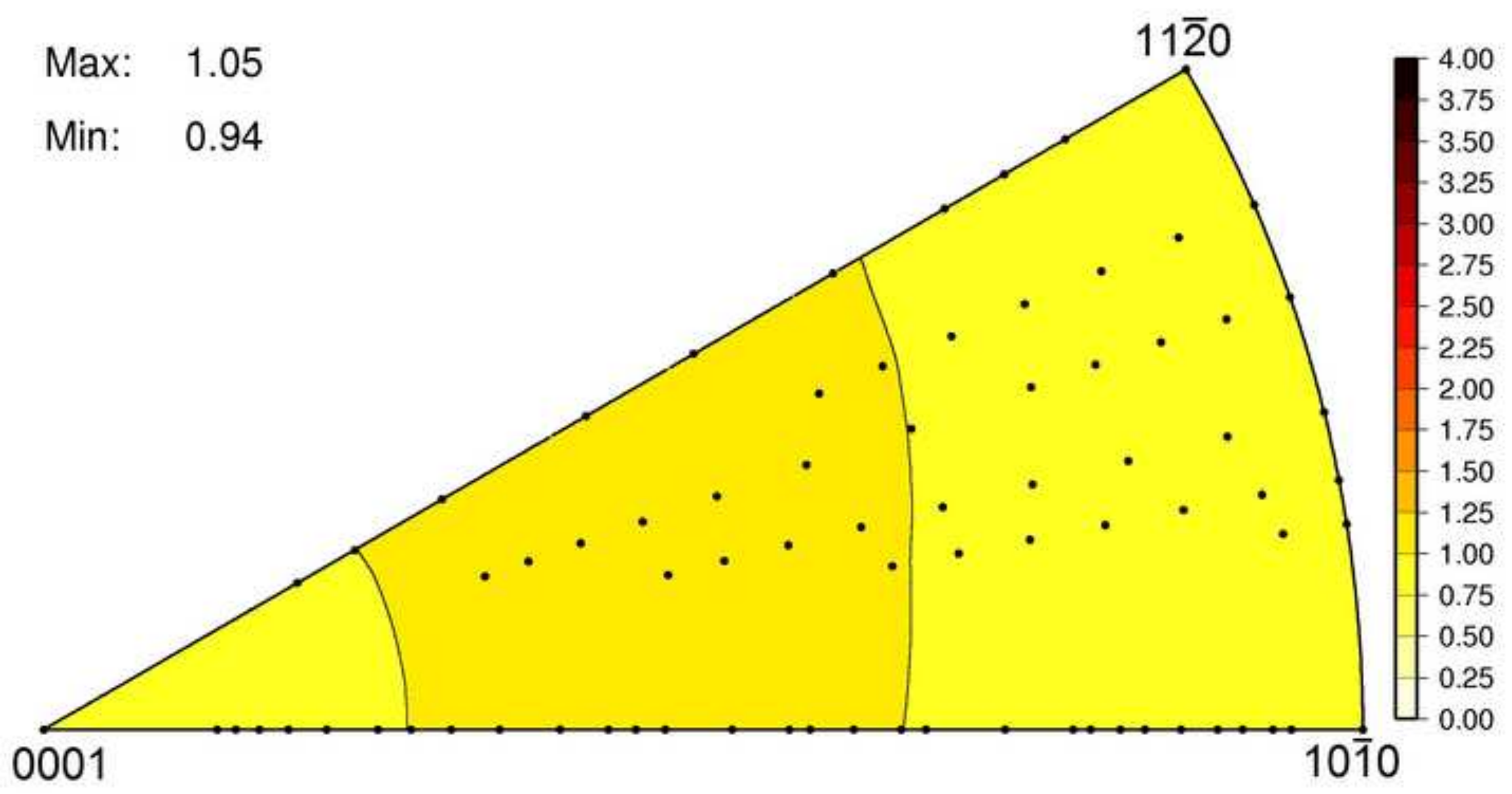




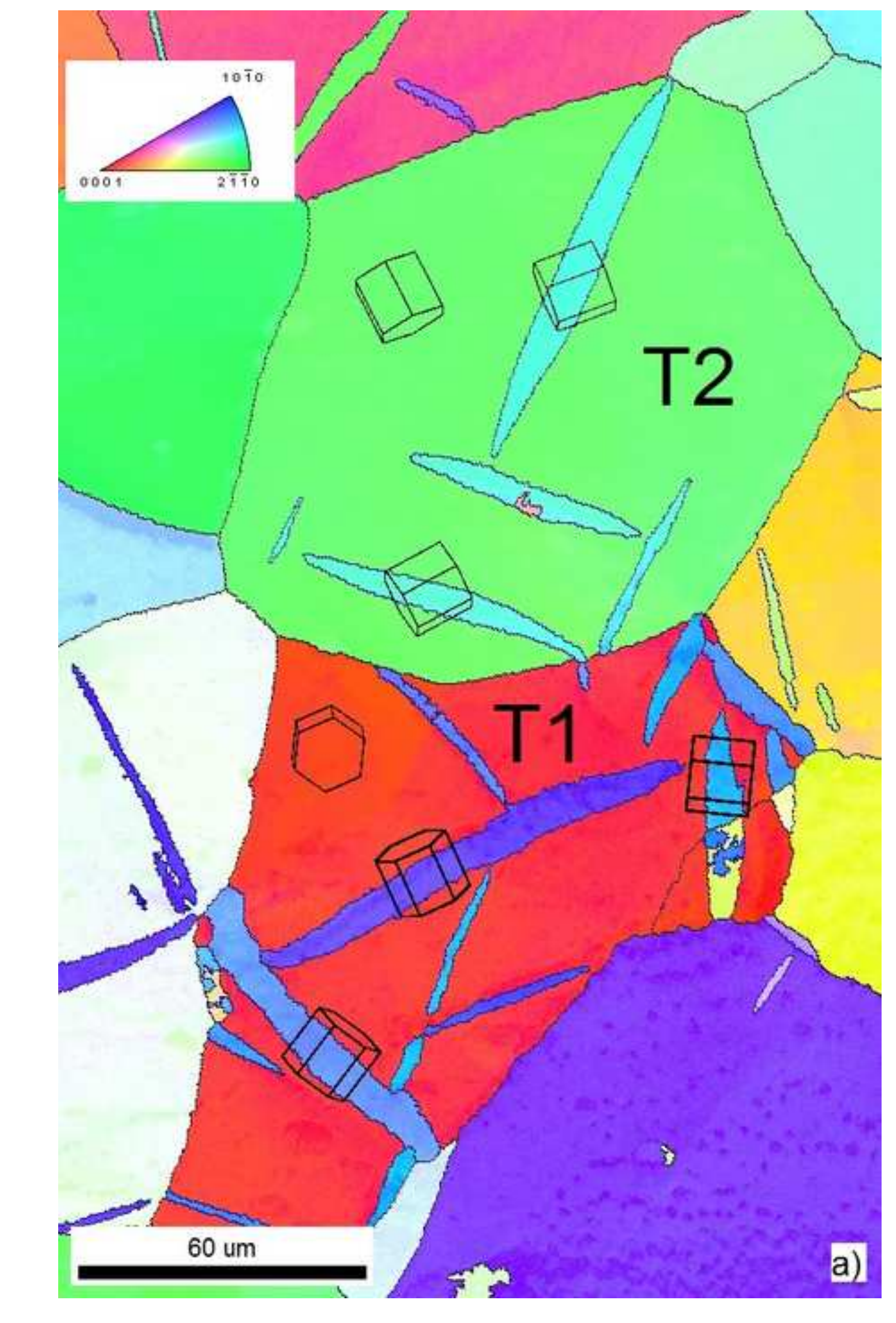

Figure 2a

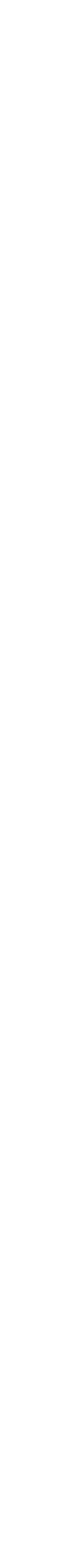

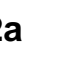




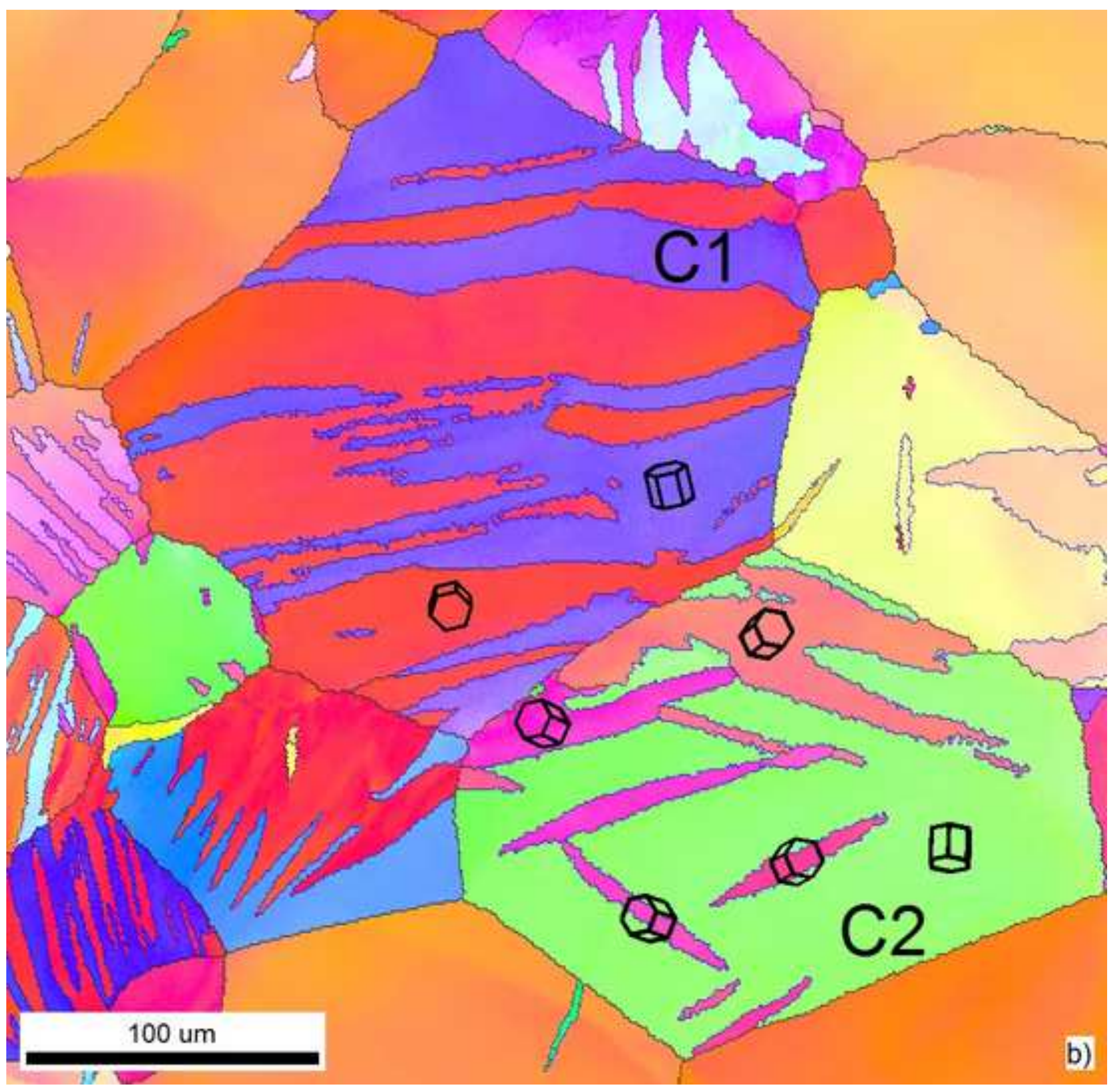




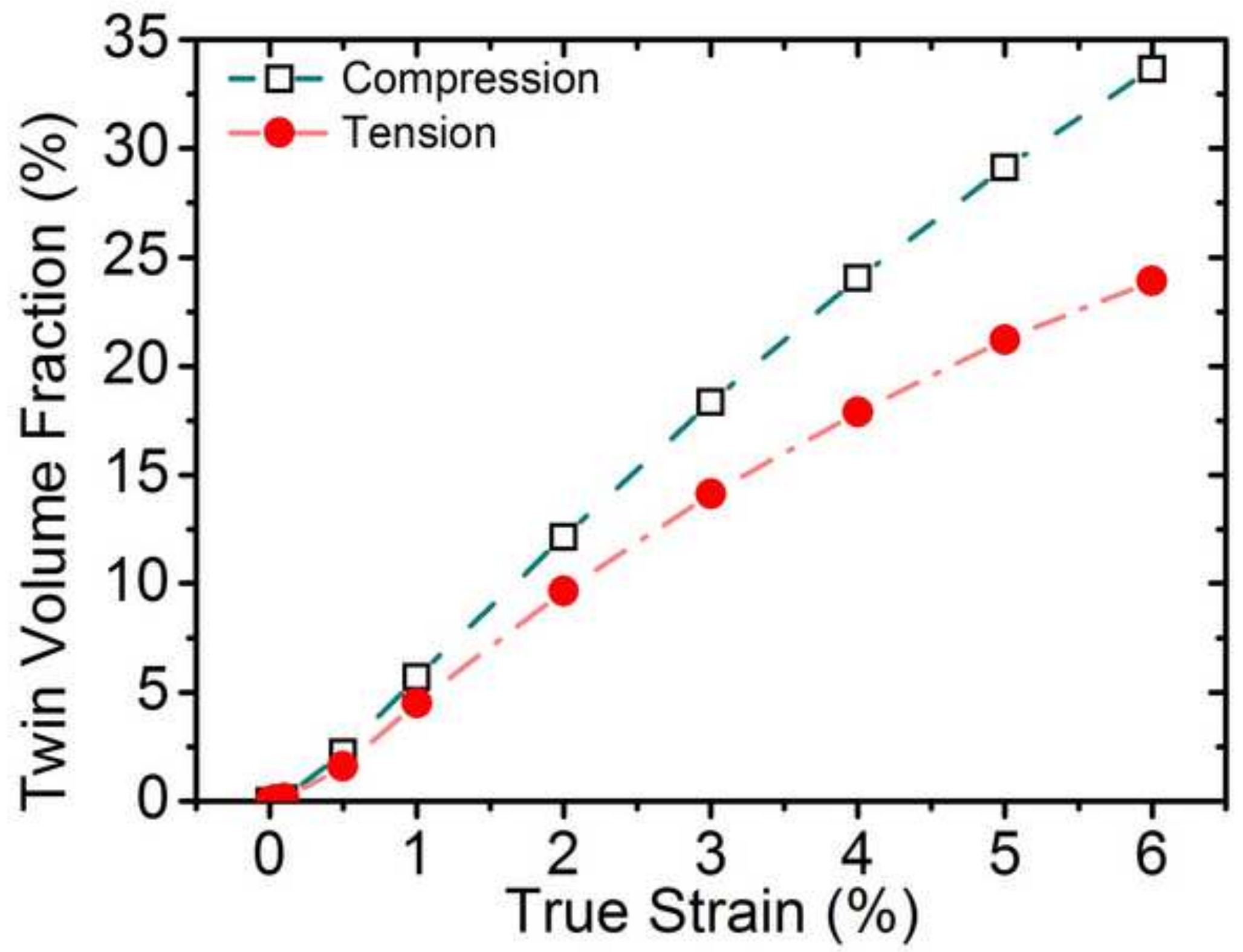




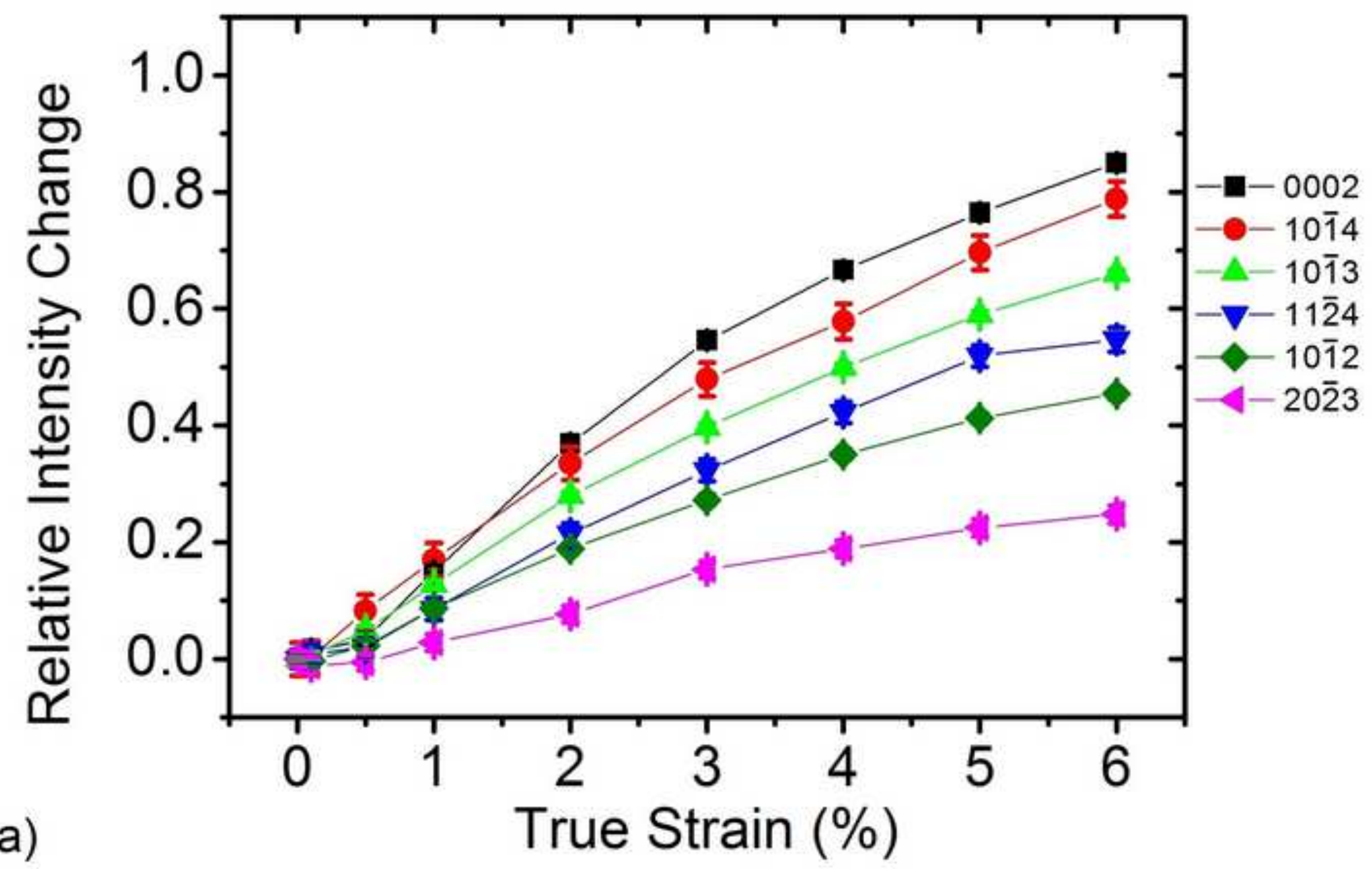




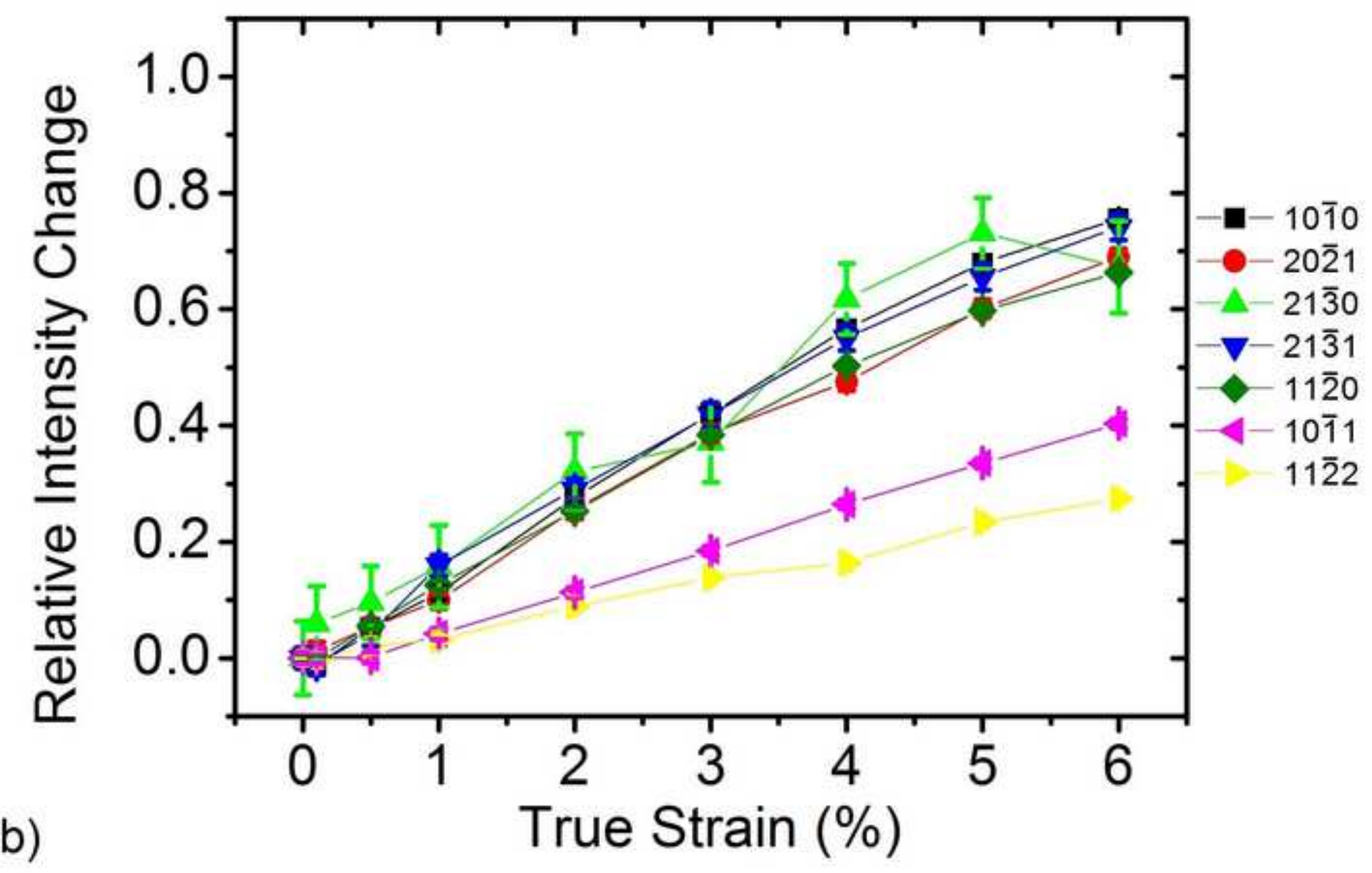

b) True Strain (\%) 


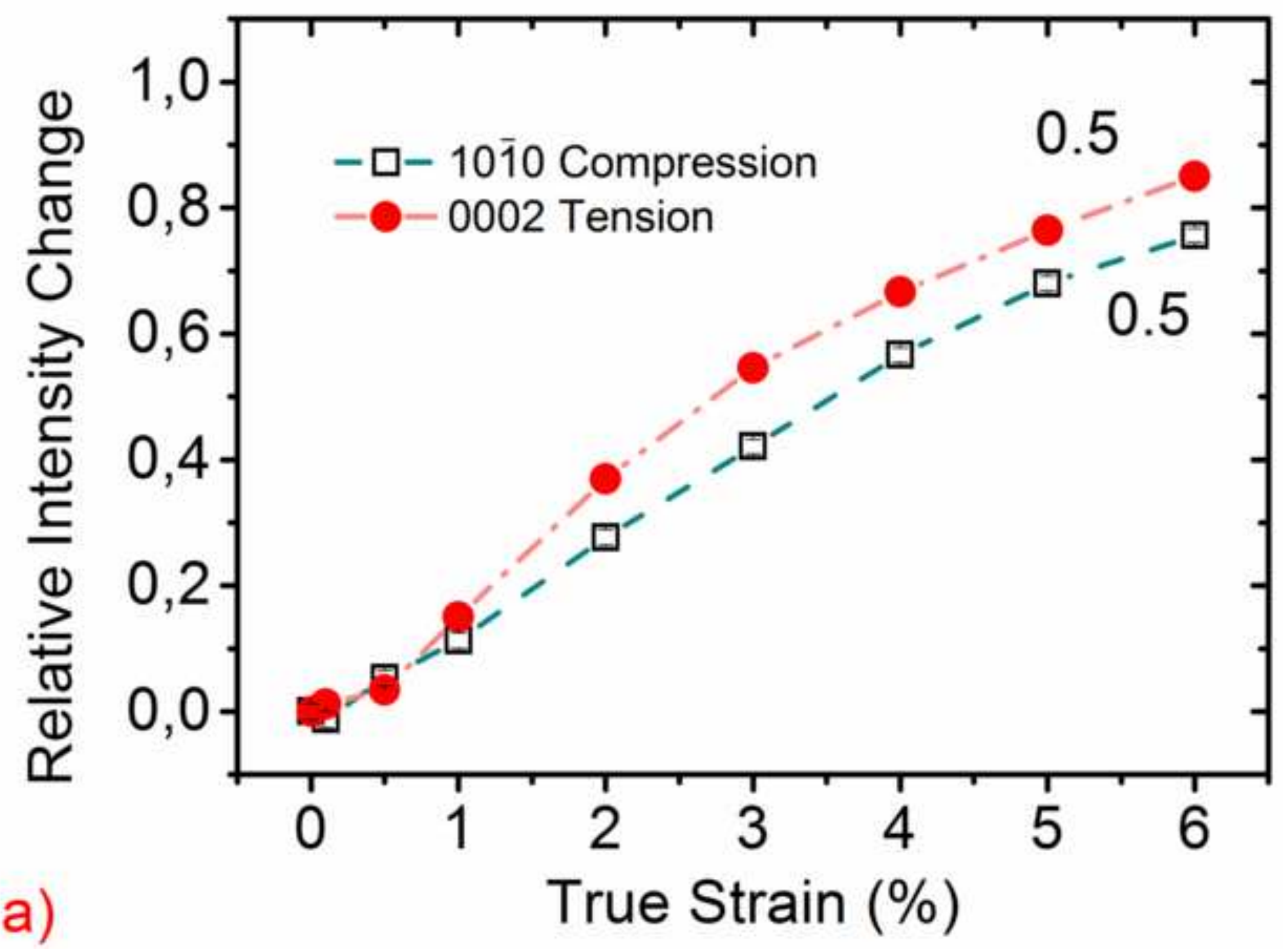

a)

True Strain (\%) 


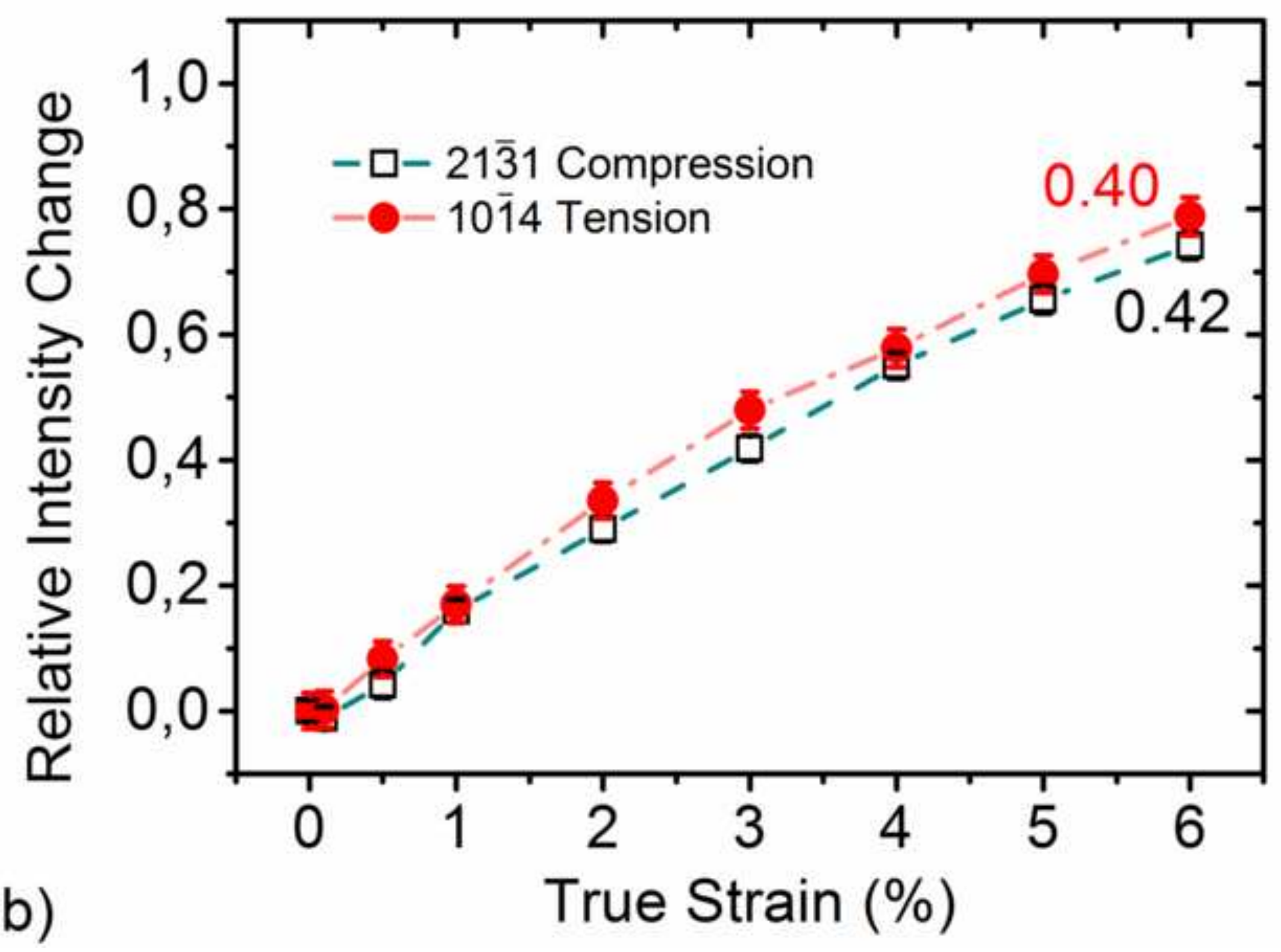

b)

True Strain (\%) 


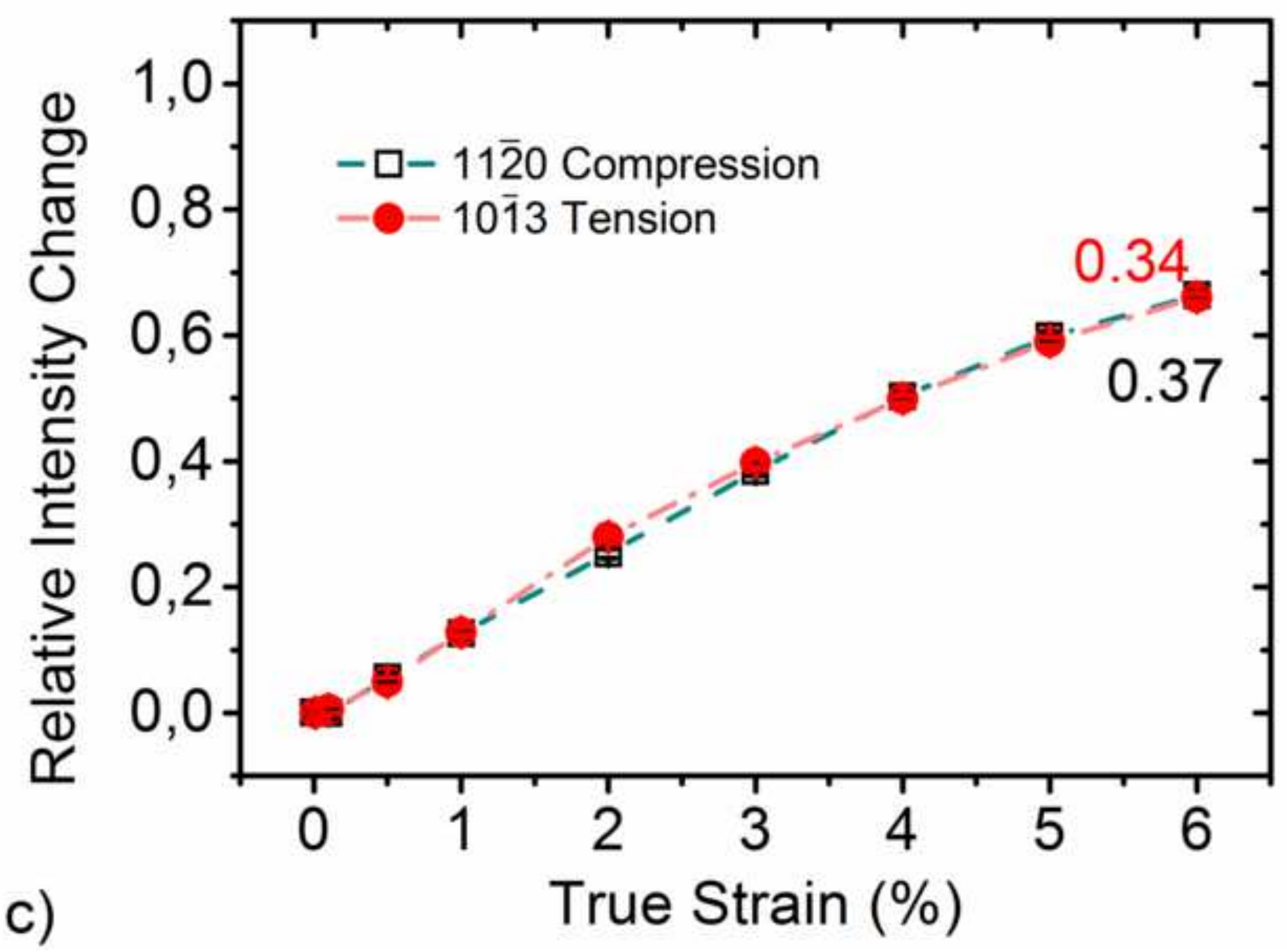

c)

True Strain (\%) 


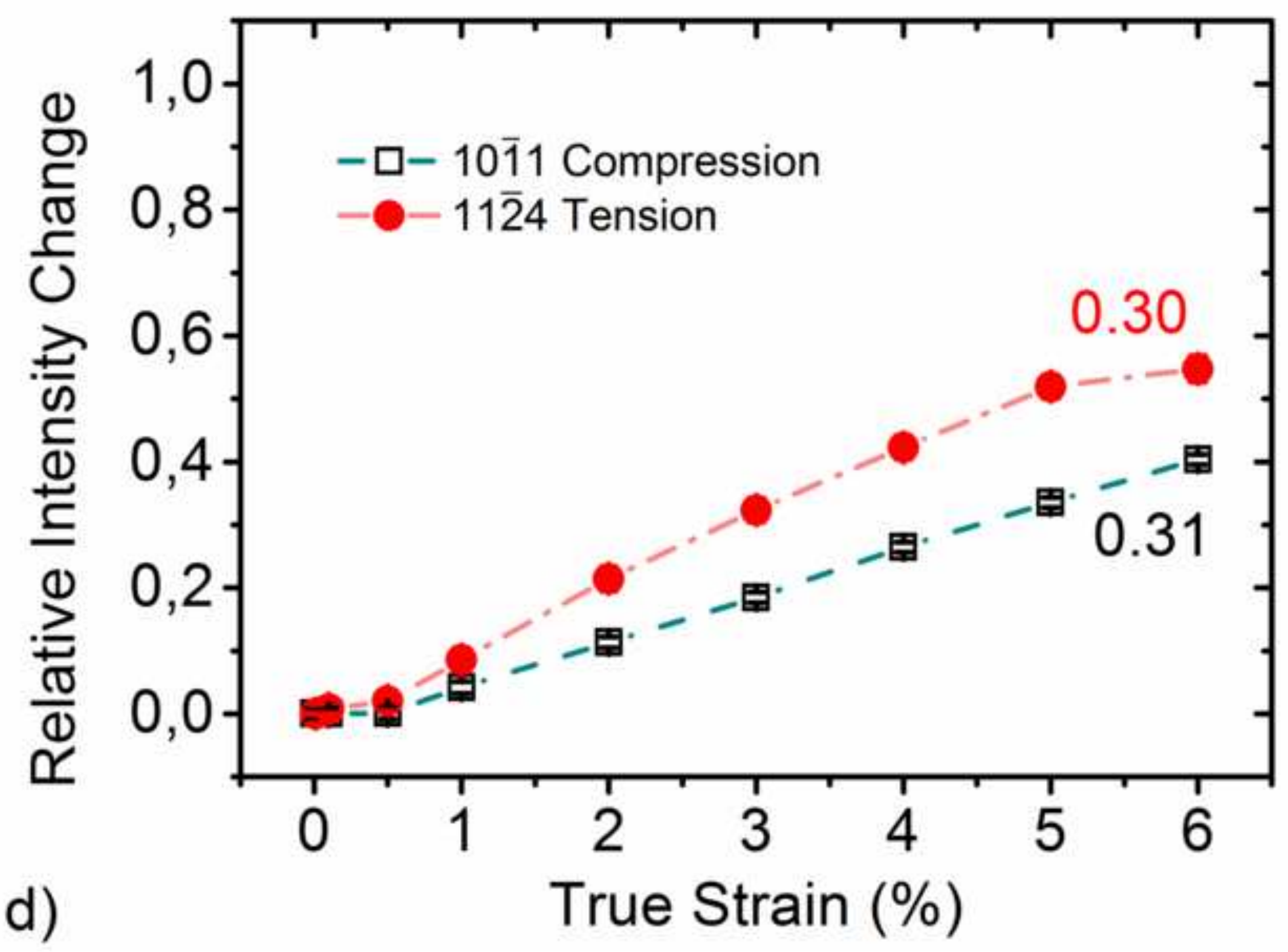

d)

True Strain (\%) 


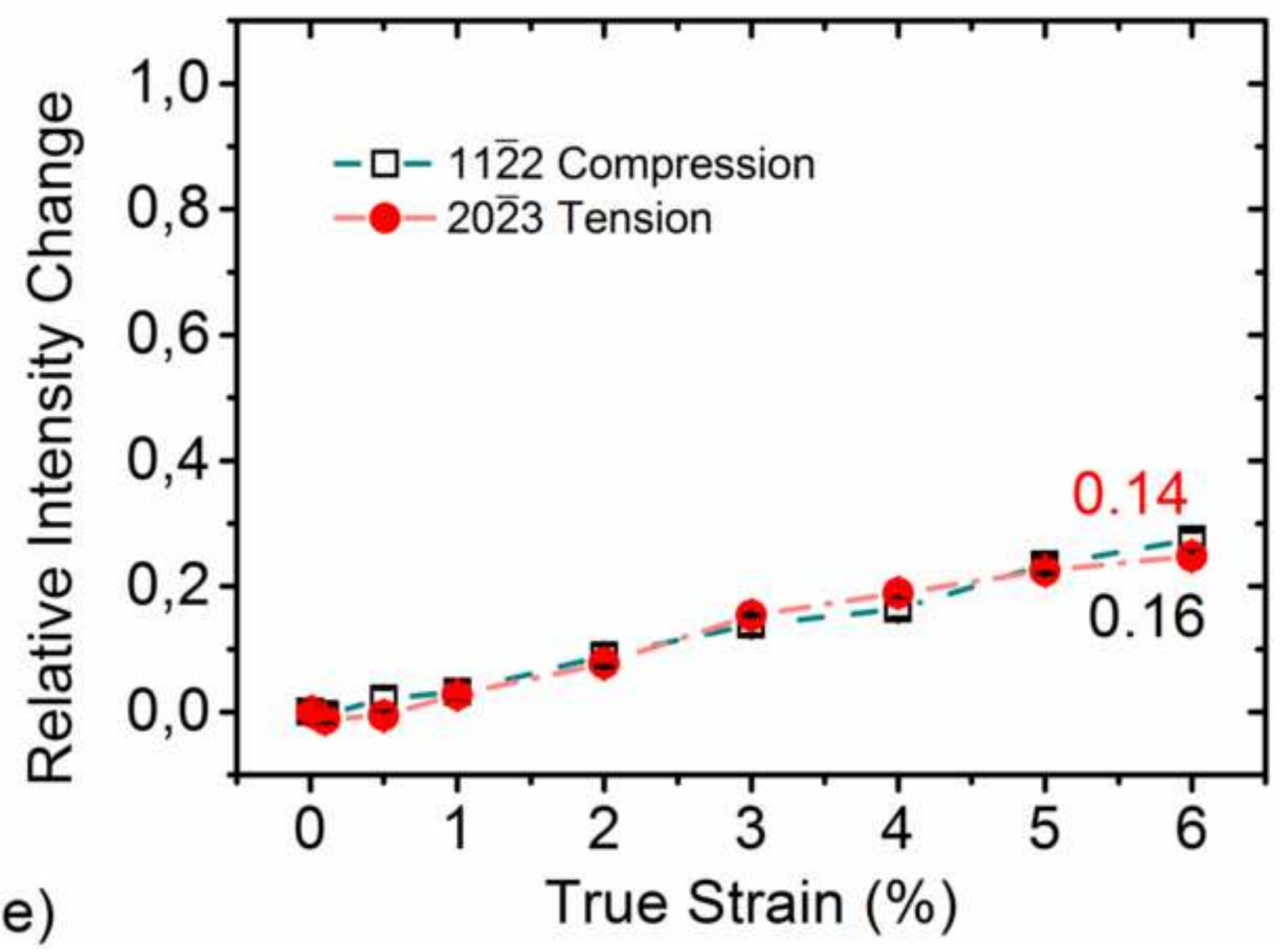

e)

True Strain (\%) 


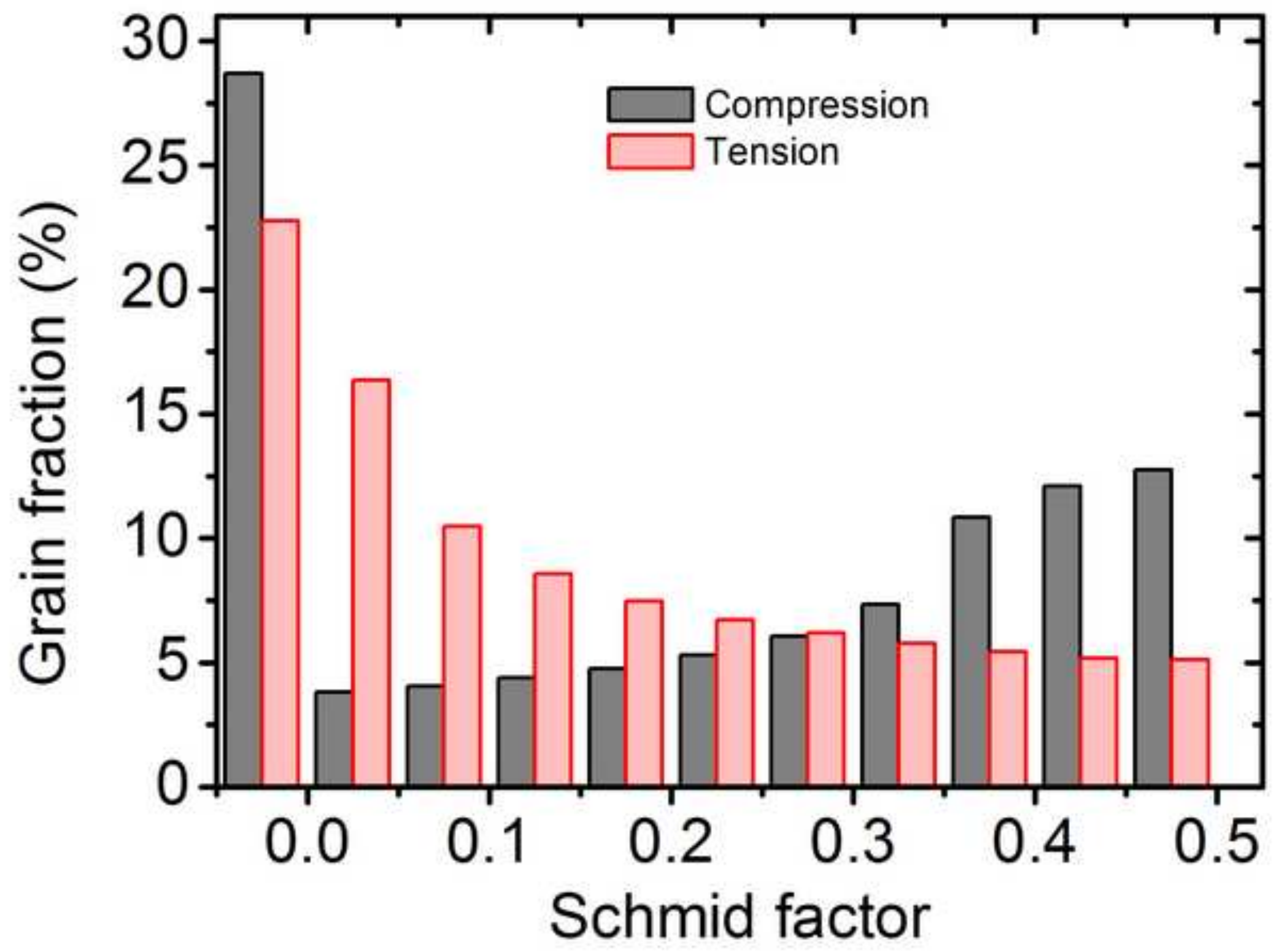




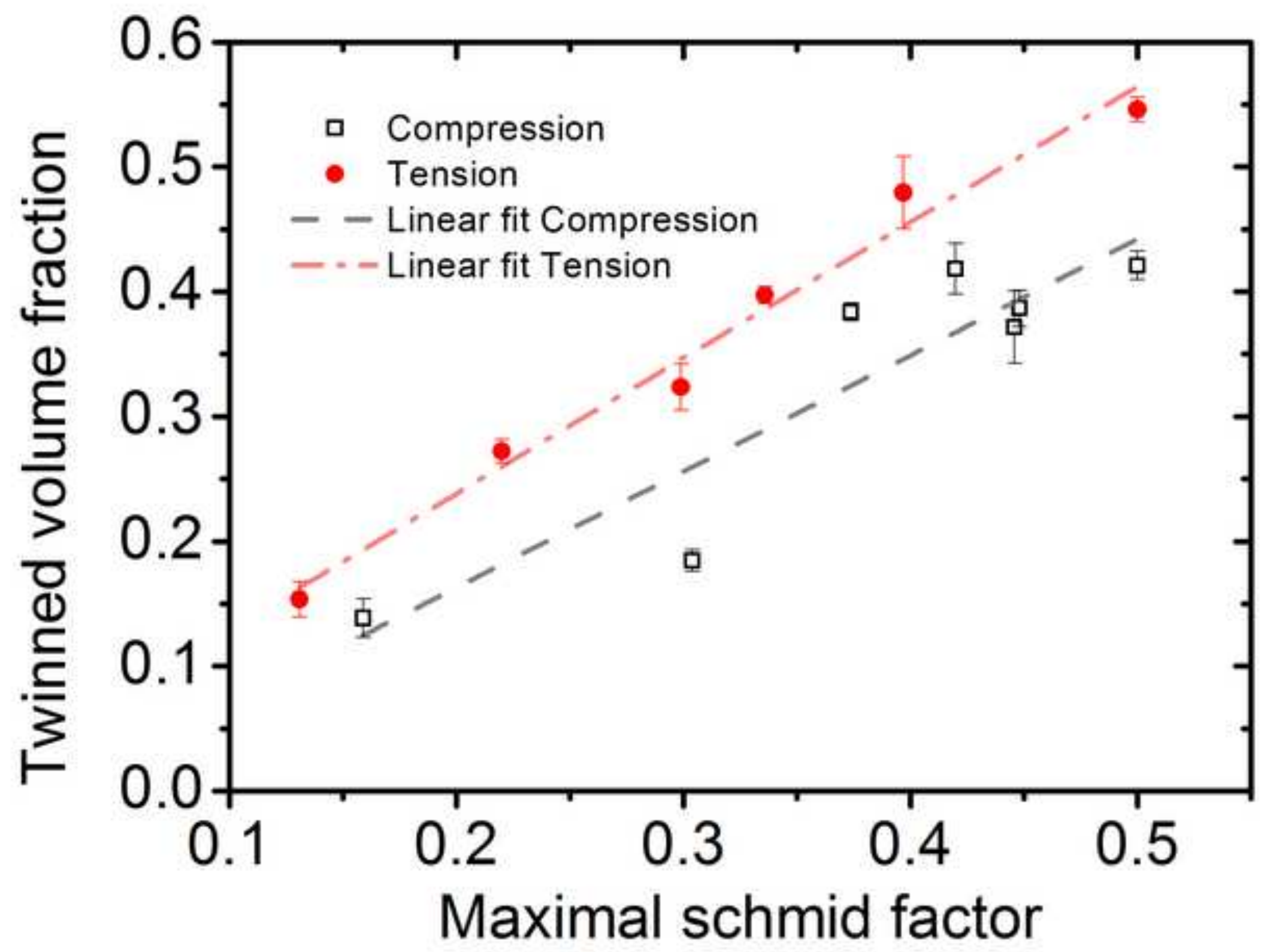




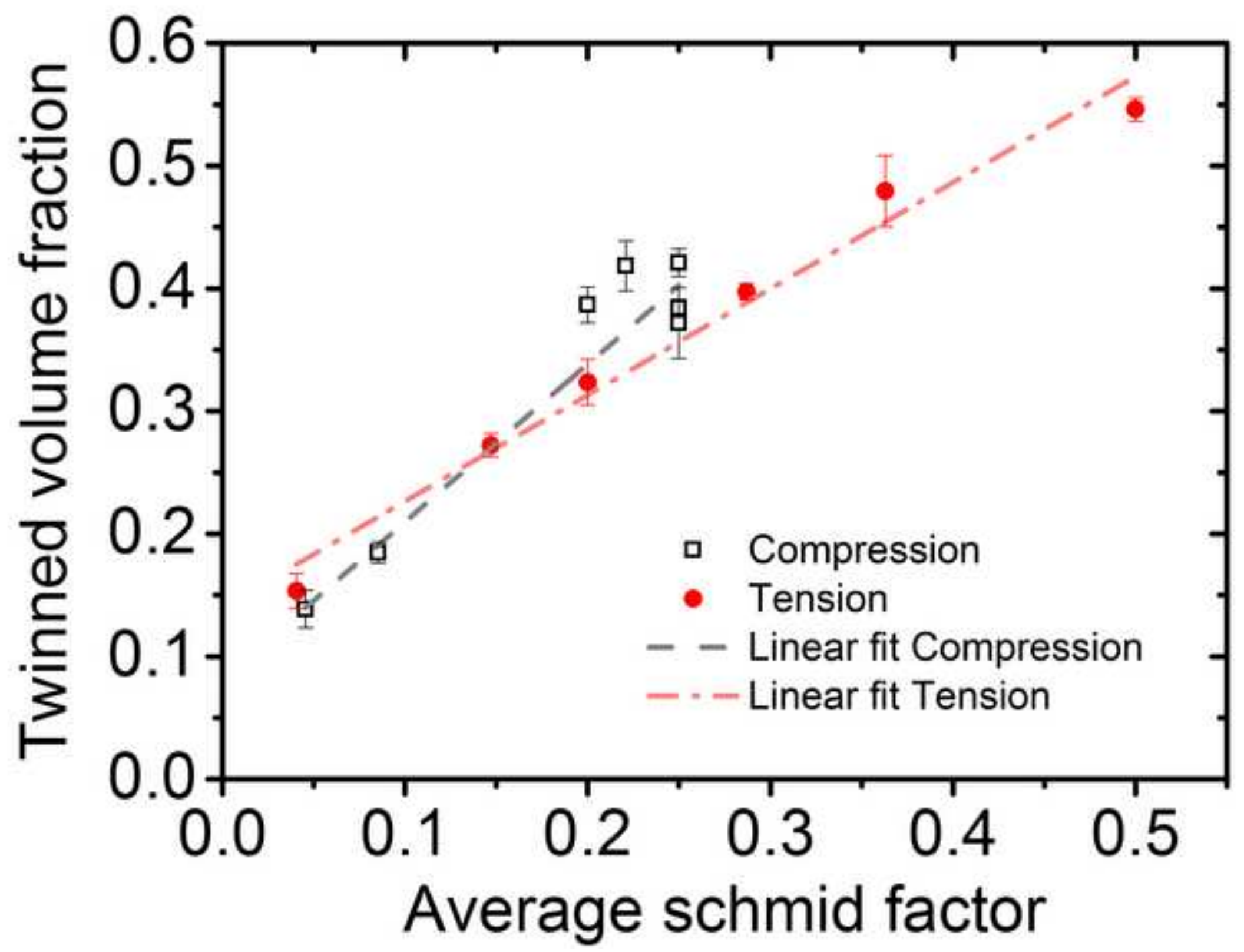




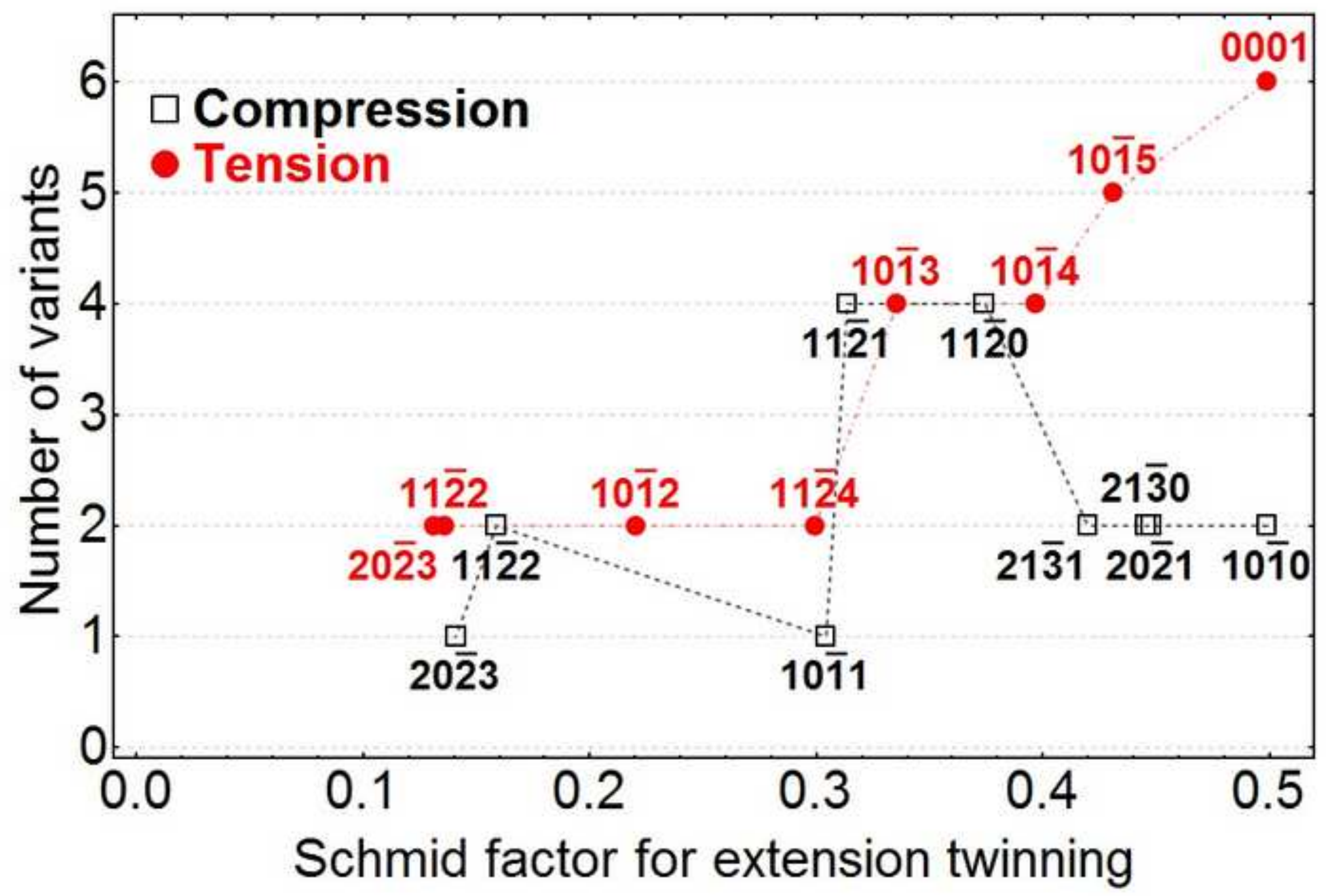




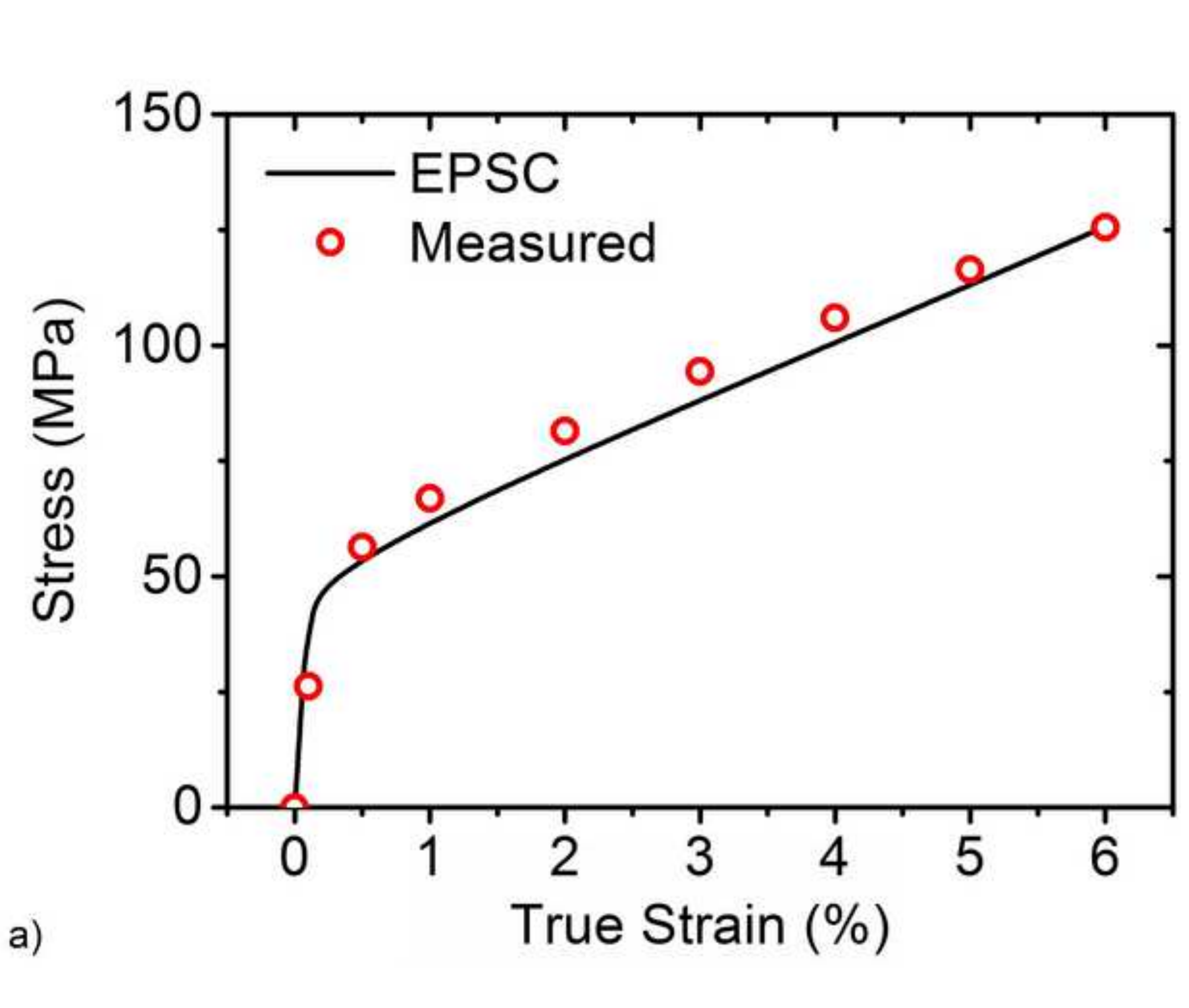

(1)

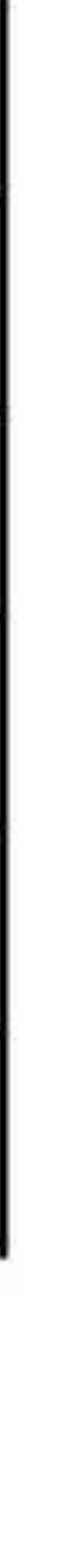

.




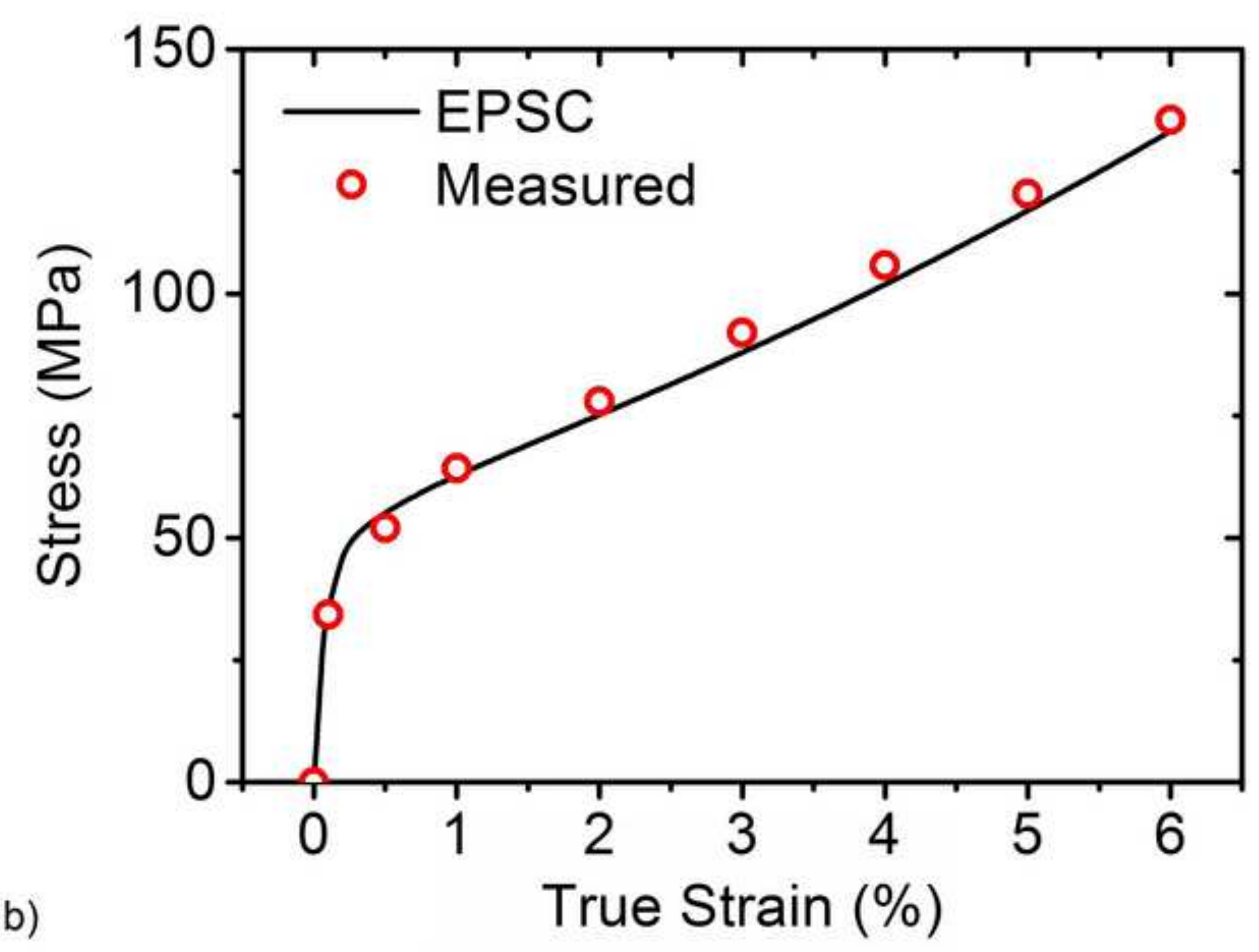

b)

True Strain (\%) 

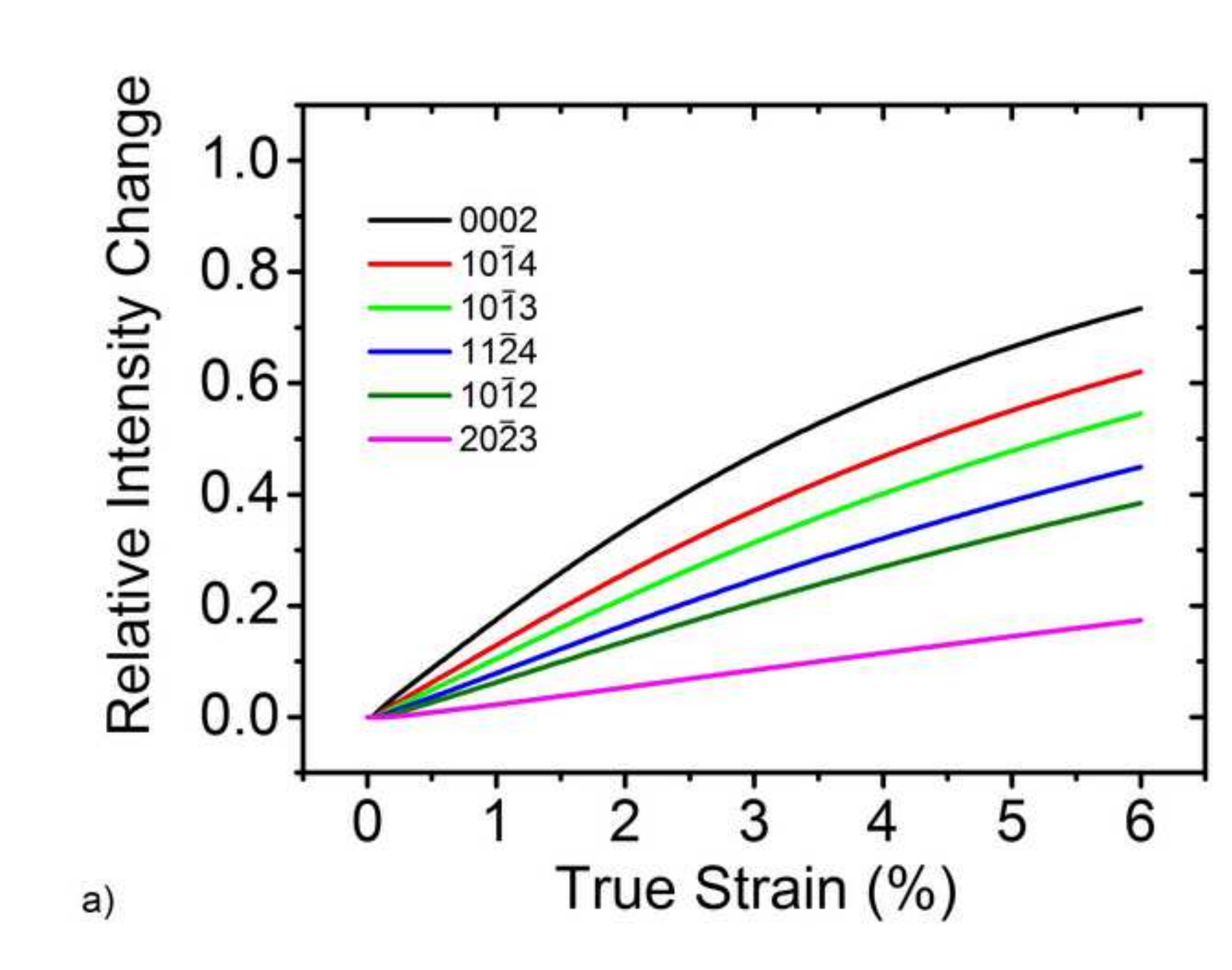

.
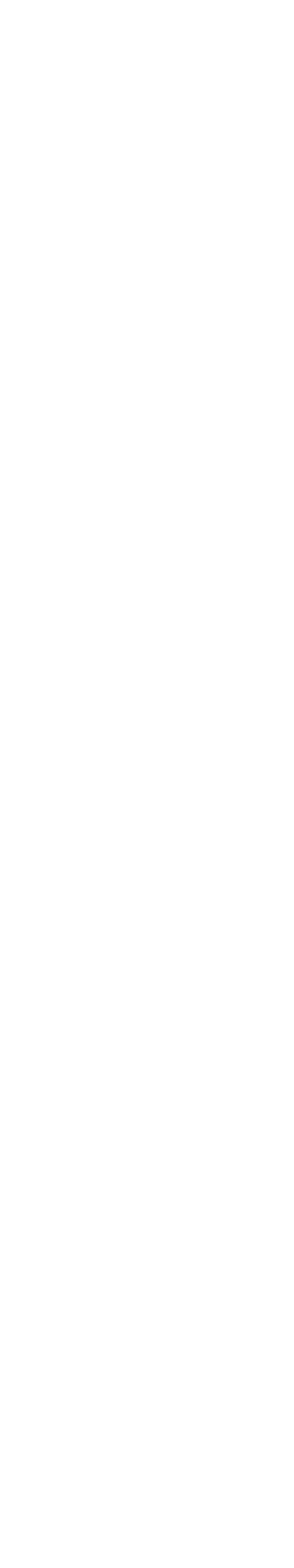

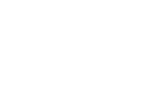

Figure 11a




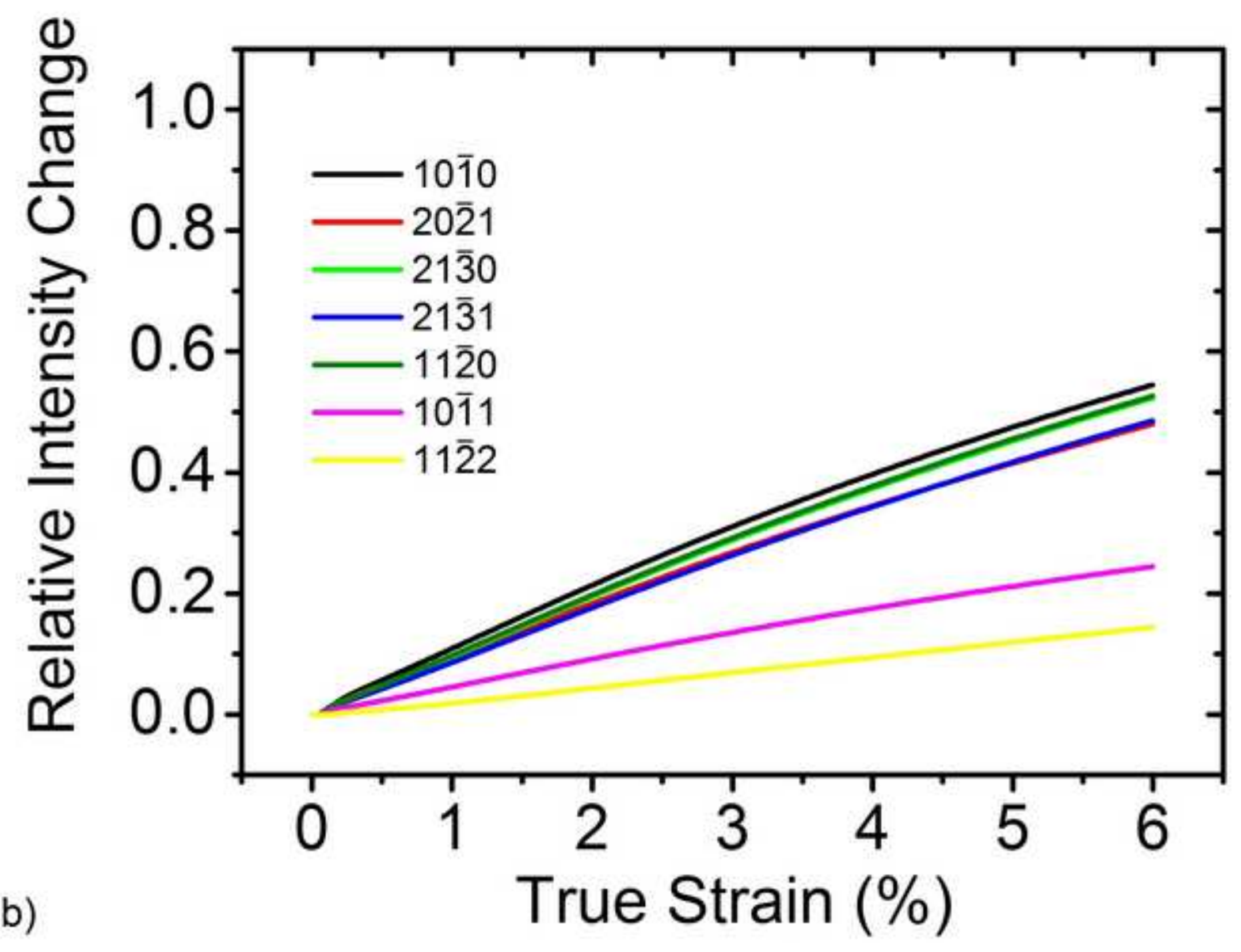




\section{Twinned grains Tension}

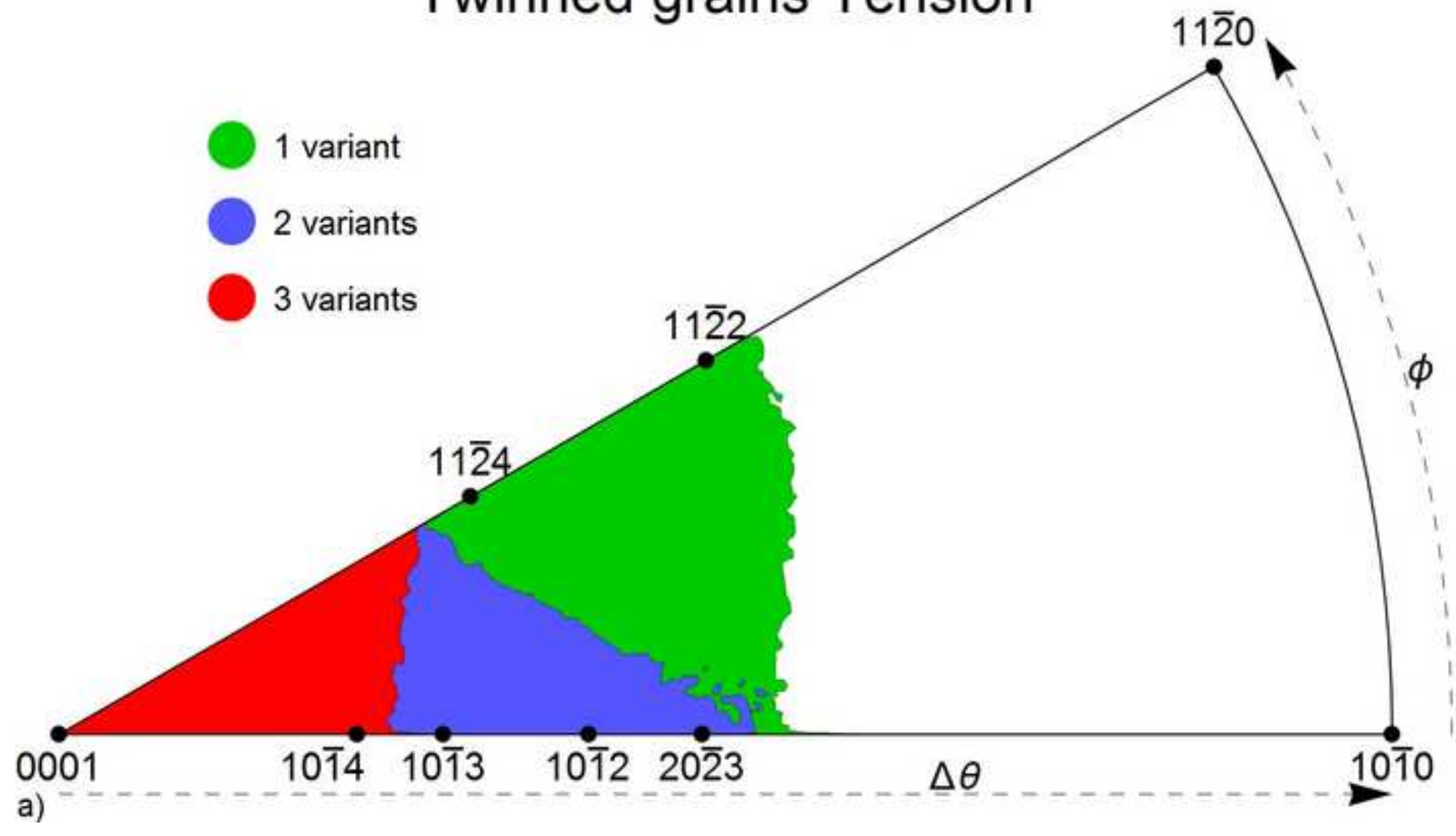




\section{Twinned grains Compression}

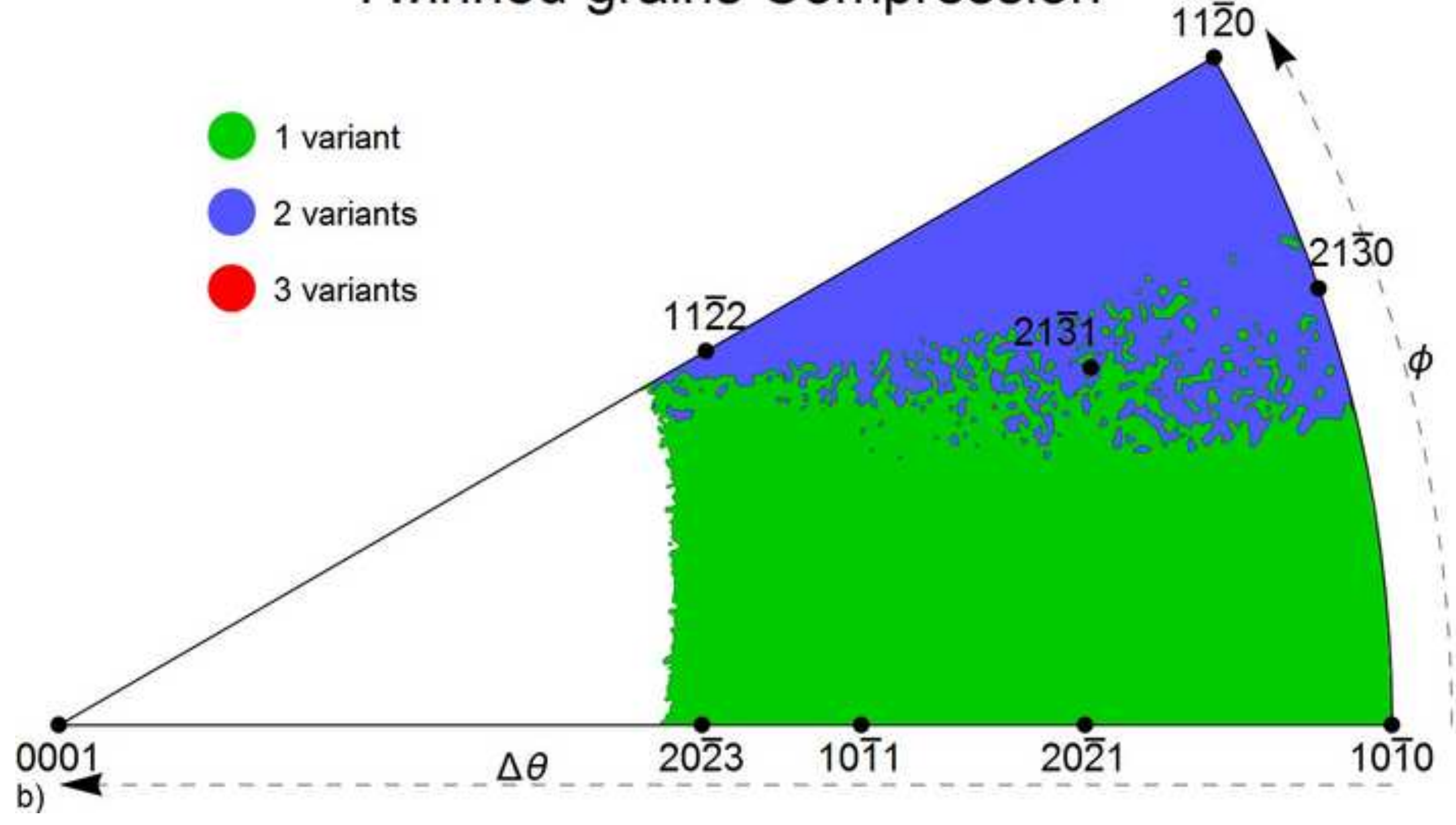



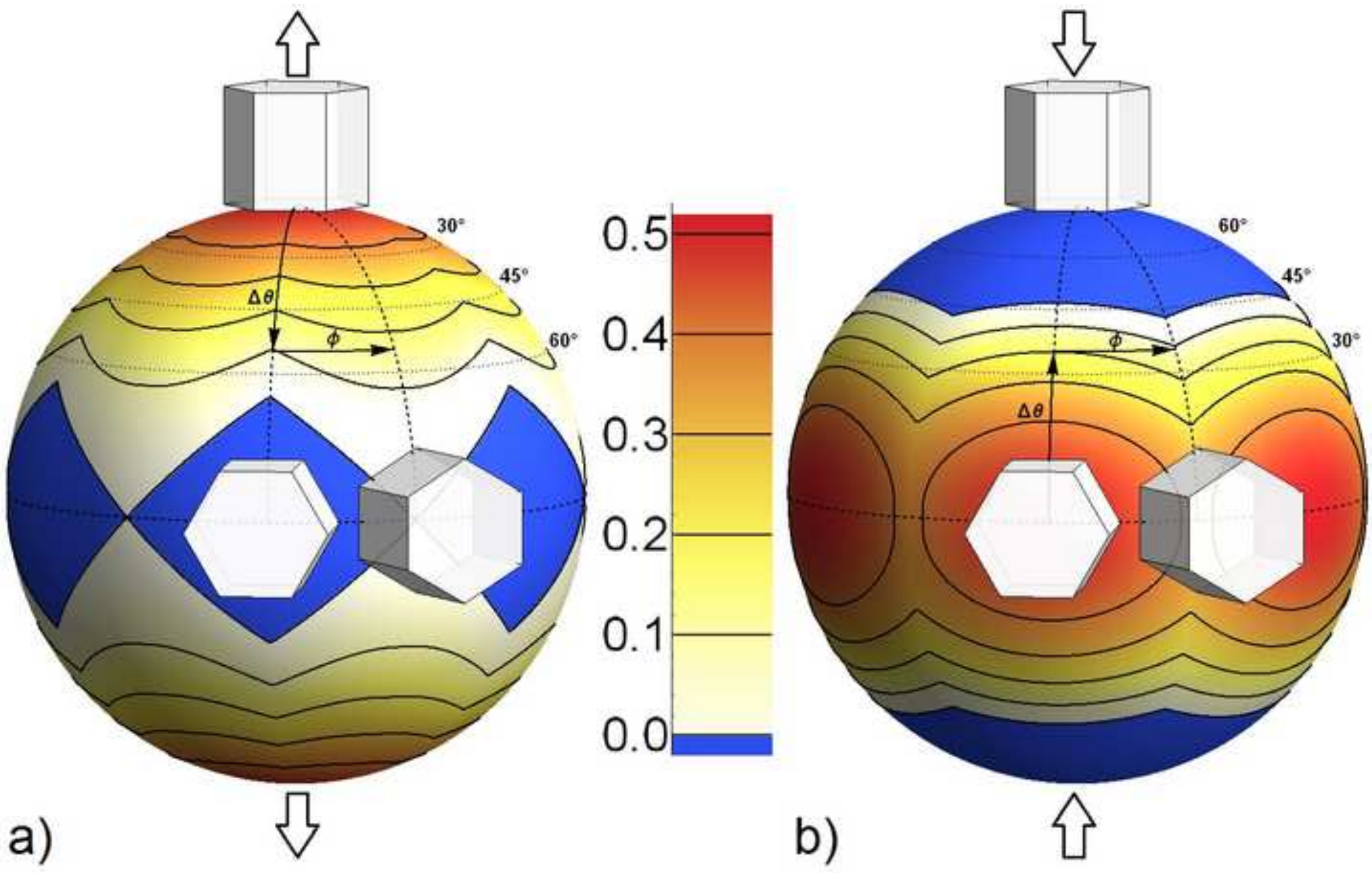


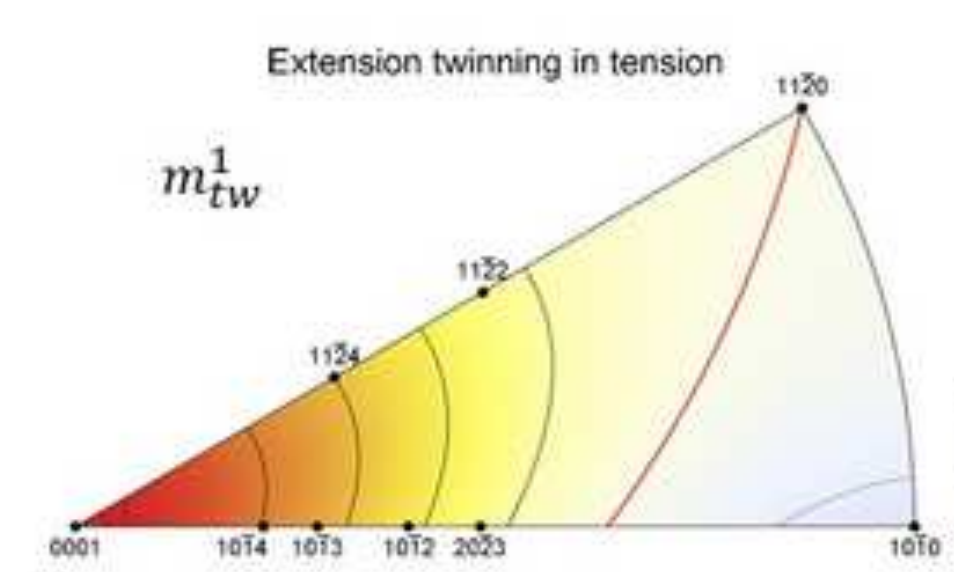

a)

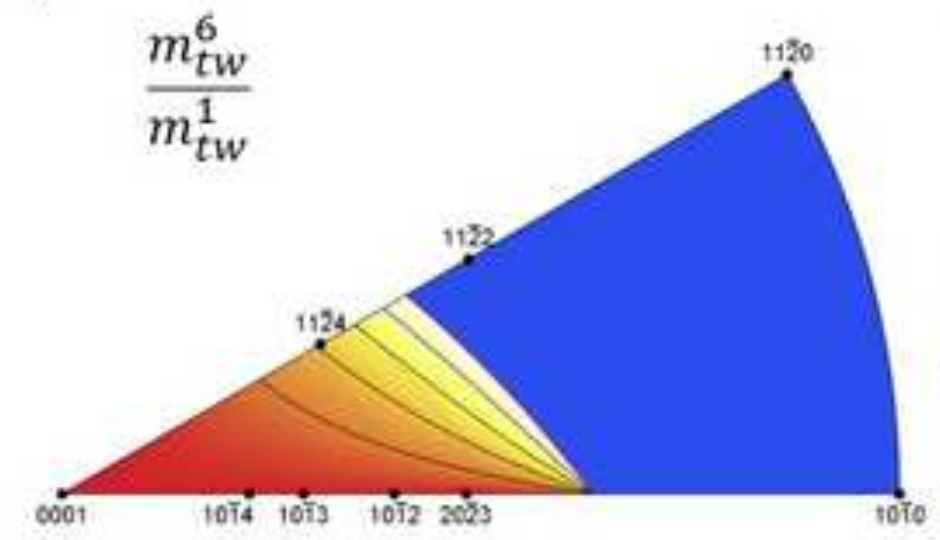

c)

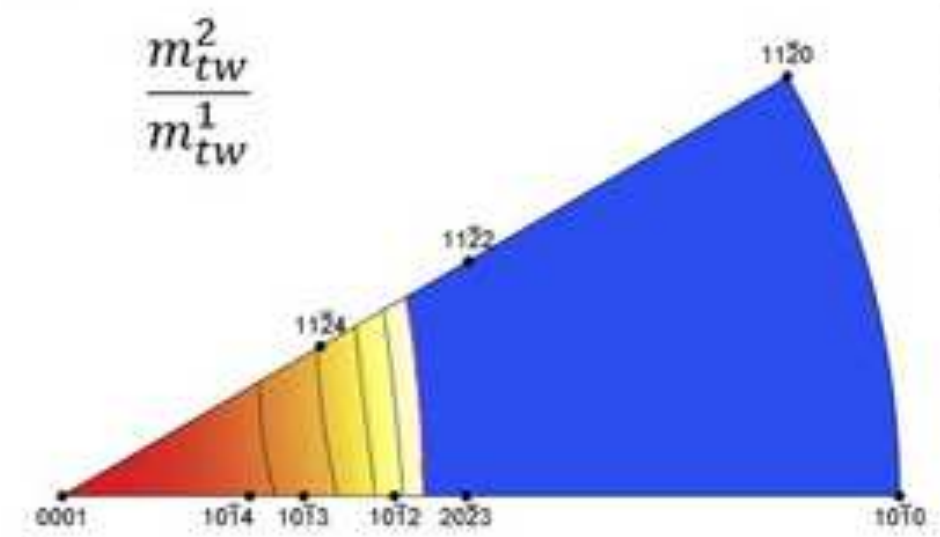

e)

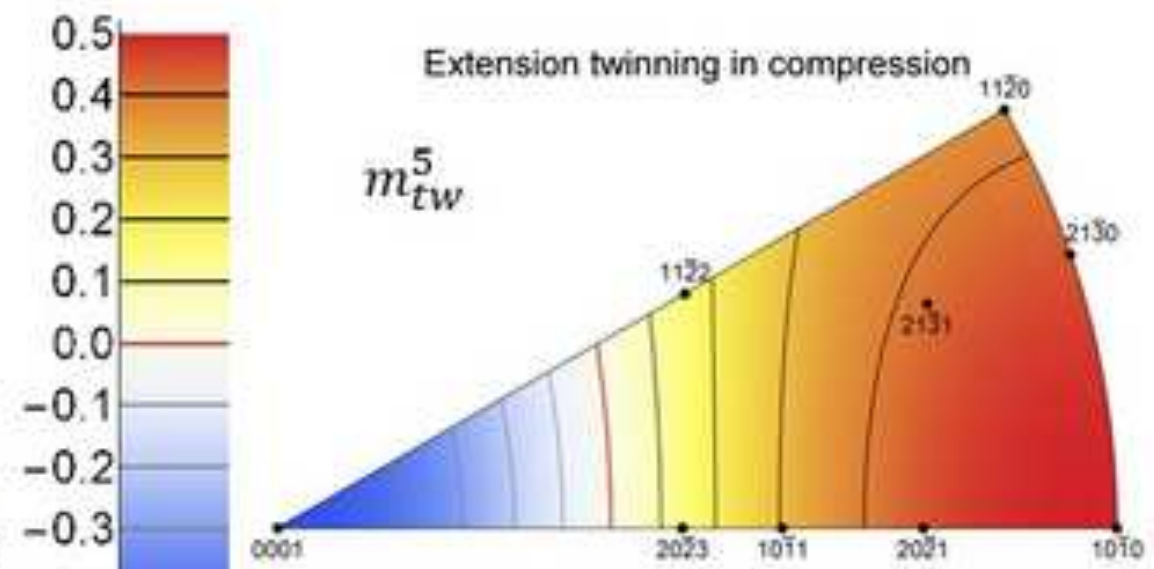

b)

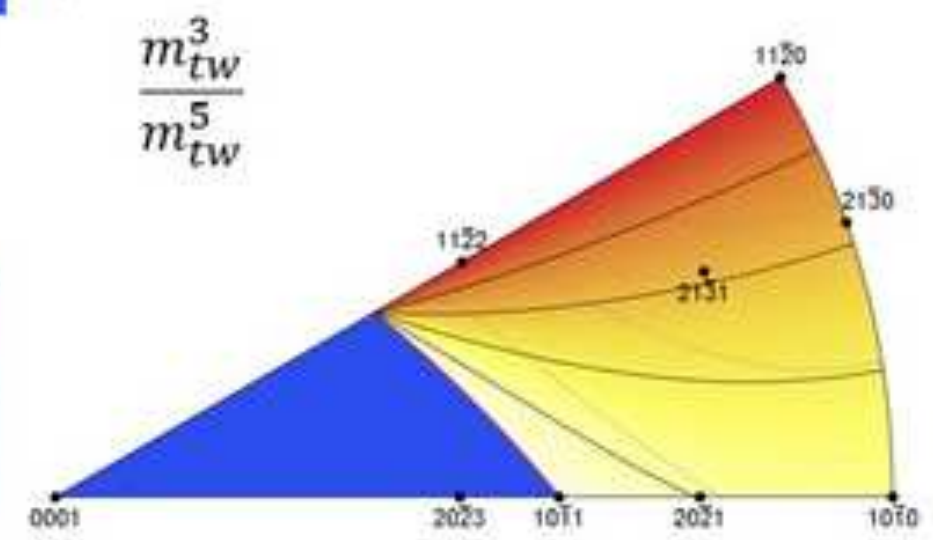

d)
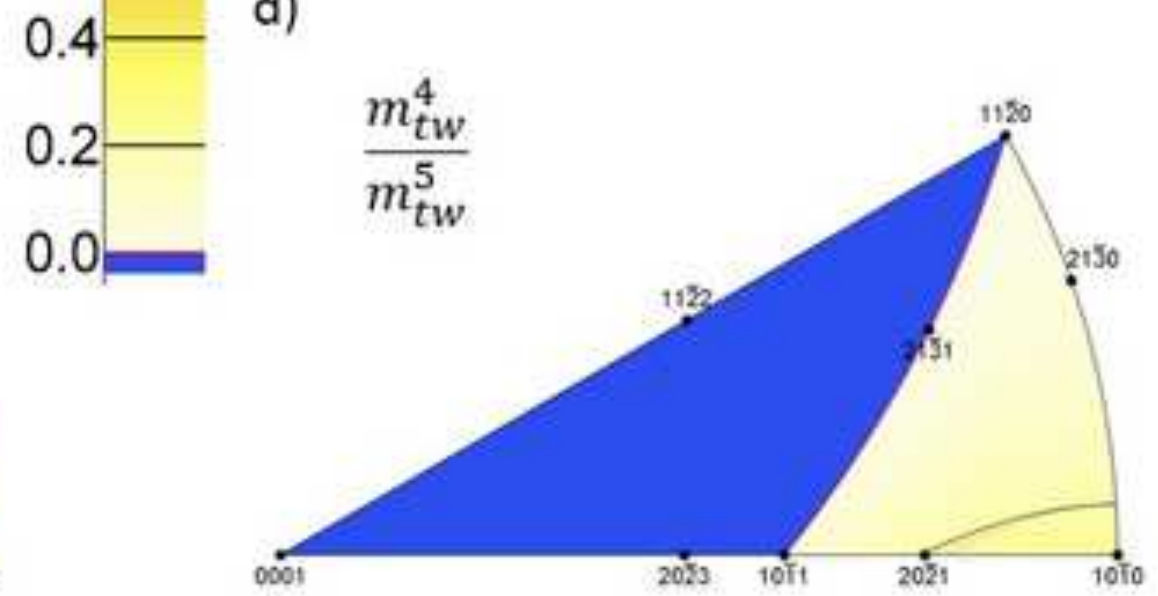

f) 


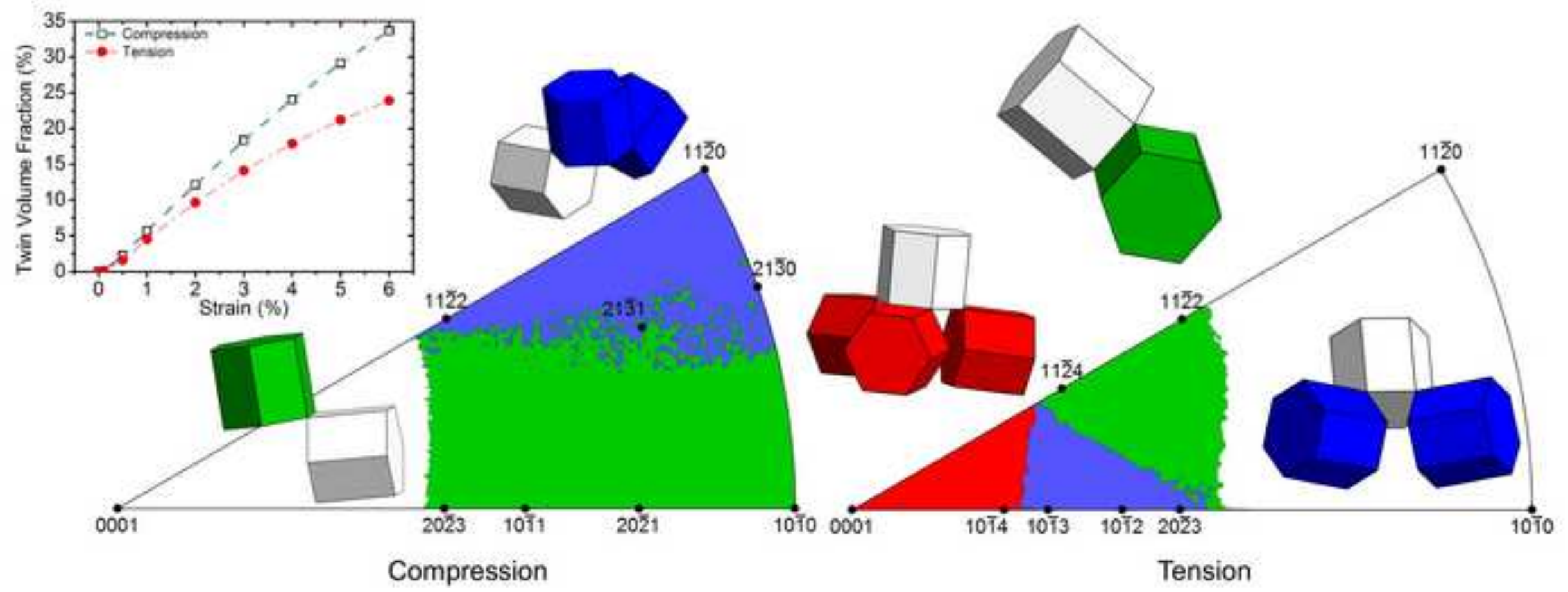

